\title{
Environ-Economic Synthesis and Characterization of Some New 1,2,4-Triazole Derivatives as Organic Fluorescent Materials and Potent Fungicidal Agents
}

\author{
Harshita Sachdeva, Rekha Saroj, Sarita Khaturia, and Diksha Dwivedi \\ Department of Chemistry, Faculty of Engineering and Technology, Mody Institute of Technology and Science, \\ Lakshmangarh, Rajasthan 332311, India
}

Correspondence should be addressed to Harshita Sachdeva; drhmsachdevaster@gmail.com

Received 6 February 2013; Revised 28 March 2013; Accepted 31 March 2013

Academic Editor: Ramadan A. Mekheimer

Copyright (C) 2013 Harshita Sachdeva et al. This is an open access article distributed under the Creative Commons Attribution License, which permits unrestricted use, distribution, and reproduction in any medium, provided the original work is properly cited.

A multicomponent one-pot clean cyclocondensation reaction of 4-chloro-2-nitro aniline/amino acids and aromatic aldehydes/ indole-2,3-diones with thiosemicarbazide in water yielding triazole/spiro indole-triazole derivatives in high yields and shorter reaction time and displaying excellent florescent property is reported. The developed MCR may provide a valuable practical tool for the synthesis of new drugs containing the title core fragment. All the newly synthesized compounds have been characterized by IR, ${ }^{1} \mathrm{HNMR},{ }^{13} \mathrm{CNMR}$, and fluorescence study and also been screened for antimicrobial activity.

\section{Introduction}

Multicomponent and domino reactions are efficient and effective methods in the sustainable and diversity-oriented synthesis of heterocycles and such reactions have attracted enormous interest in recent years [1]. Thiosemicarbazide and its derivatives are an important class of synthetic compounds, having large variety of applications due to their wide spectrum of biological activities [2] including antiviral [3] and antitumoral [4] as well as parasiticidal activity against Plasmodium falciparum, Plasmodium berghei [5], Trypanosoma cruzi [6-8] Trypanosoma brucei rhodesiense [9], and Toxoplasma gondii [10].

The 1,2,4-triazoles and their derivatives are found to be associated with various biological activities such as anticonvulsant [11], antifungal [12], anticancer [13], antiinflammatory [14], and antibacterial properties [15]. Also several compounds containing 1,2,4-triazole rings are well known as drugs; for example, fluconazole is used as an antimicrobial drug, while vorozole, letrozole, and anastrozole are nonsteroidal drugs used for the treatment of cancer.

The increasing diversity of small molecule libraries is an important source for the discovery of new drug candidates.
In terms of this trend, the literature survey showed that indole derivatives possess anticancer [16, 17], antioxidant [18], antibacterial [19], antifungal [20, 21], antiviral [22, 23], and antihypertensive activities [24]. Indole-3-carbon atom in the form of spiro carbon atom exhibits enhanced biological activities $[25,26]$.

The important biological activities of triazole derivatives impelled us to take up the synthesis of these new combinational heterocycles which are likely to have augmented diverse biological activity. The developed MCR may provide a valuable practical tool for the synthesis of novel physiologically active agents containing the title core fragment. Several methods [27-30] for the synthesis of 1,2,4-triazole derivatives are reported in the literature but all these methods require the presence of organic solvents and also require long reaction time. Further, there are only few reports [31-33] available in the literature regarding the synthesis of triazole derivatives under environmentally benign conditions. Earlier we also reported [33] the synthesis of spiro indole-triazoles under microwaves using montmorillonite as an inorganic solid support.

In recent years, organic research is mainly focused on the development of greener and environ-economic methods 


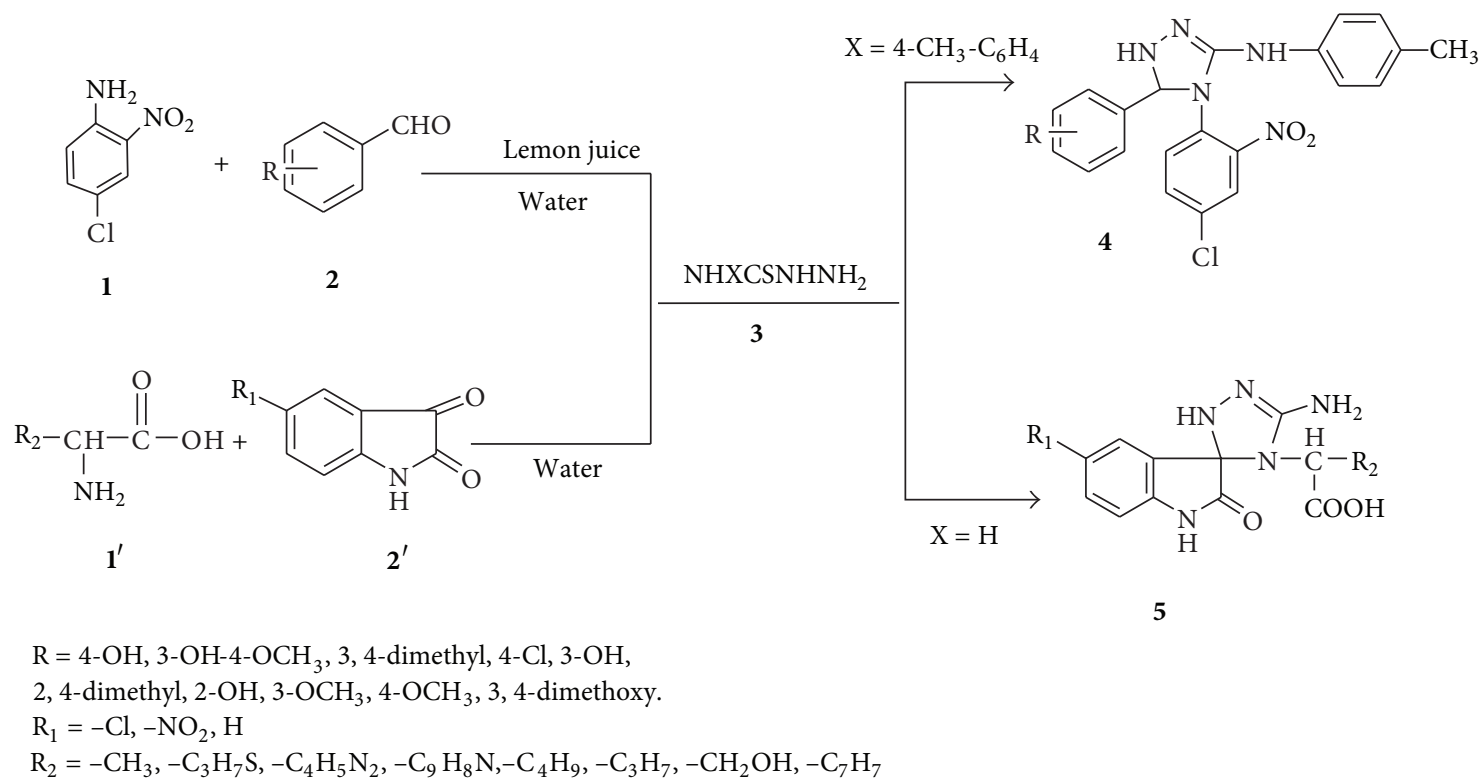

Scheme 1: Synthesis of triazole derivatives 4 and 5.

which involve use of alternative reaction media to replace volatile and hazardous solvents like benzene, toluene, and methanol, commonly used in organic synthesis. Nowadays, many organic transformations have been carried out in water [34-38]. It is a unique solvent because it is readily available, inexpensive, nontoxic, safer, and environmentally benign.

Recently, triazole-based fluorescent sensors have been developed for the selective detection of platinum ions in aqueous solutions [38] and synthesis of a pyrenyl-appended triazole-based calyx[4] arene [39] as a fluorescent sensor for $\mathrm{Cd}^{+2}$ and $\mathrm{Zn}^{+2}$ is carried out. Prompted by rapidly expanding applications of organic fluorescent materials for electroluminescence (EL), dyelasers, sensors, probes, and phototherapeutic agents, and in view of the florescent properties associated with triazole derivatives, development of new fluorescent organic compounds with high functionality has been the subject of intense study for more than a decade [40-43].

In this context, we have synthesized new 1,2,4-triazole derivatives (4/5) using water as a green solvent under the umbrella of green chemistry and synthesized compounds have been evaluated for antibacterial and antifungal activity and also fluorescent properties.

\section{Result and Discussion}

In continuation of our work to develop greener and expeditious protocols for the synthesis of heterocyclic compounds [44-48], herein we wish to report a highly efficient procedure for the synthesis of new substituted-2H-1,2,4-triazole phenol derivatives (4) and spiro (indole-triazole) propanoic acid derivatives (5) using water (containing 1-2 mL of alcohol) by the reaction of 4 -chloro-2-nitro aniline (1)/amino acids $\left(\mathbf{1}^{\prime}\right)$ and aromatic aldehydes $(2) / 1 \mathrm{H}$ indole-2,3-diones $\left(\mathbf{2}^{\prime}\right)$, respectively, with thiosemicarbazide (3) yielding triazole (4)/spiro indole-triazole (5) derivatives in high yields and shorter reaction displaying excellent florescent property (Scheme 1).

One of the tools used to combine economic aspect of new reactions with environmental aspects is the multicomponent reaction strategy. Compound $\mathbf{4}$ was synthesized by the onepot multicomponent reaction of $\mathbf{1 , 2}$, and 3 in the presence of lemon juice as a natural acidic catalyst in aqueous medium. There are few reports $[49,50]$ of synthesis of heterocyclic compounds using lemon juice. For the present work, we have used extract of Citrus limonum species of lemon as natural catalyst for the synthesis of triazole derivatives. The main ingredients of lemon juice are moisture (85\%), carbohydrates $(11.2 \%)$, citric acid (5-7\%), protein (1\%), vitamin-C (0.5\%), fat $(0.9 \%)$, minerals $(0.3 \%)$, fibers $(1.6 \%)$, and some other organic acids. As lemon juice is acidic in nature $(\mathrm{pH} \approx 2$ 3 ) and percentage of citric acid (5-7\%) is more than other acids, it works as acid catalyst [49] for synthesis of triazole derivatives. Using this methodology these reactions were completed in shorter reaction times (1-2 hrs) with yields of the product ranging from 78 to $83 \%$. To our satisfaction, we found that the use of $2 \mathrm{~mL}$ of lemon juice resulted in quantitative yield of the corresponding triazole derivatives (4) within 1 to 2 hrs.

Mechanism of formation of compound 4 involves acidcatalyzed nucleophilic attack of 4-chloro-2-nitro aniline [1] on carbonyl carbon [7] forming arylimino derivatives preferentially [11]. The nucleophilic attack of the amino electrons of thiosemicarbazide at the electrophilic benzylidene carbon [12] accompanied by the migration of a hydrogen atom forms an intermediate thiol derivative [13] which on cyclisation and desulfurization with the loss of $\mathrm{H}_{2} \mathrm{~S}$ gives the suggested triazole derivatives (4) (Scheme 2).

On the other hand, synthesis of spiro indole triazole (5) was carried out in the absence of lemon juice by the reaction 
<smiles>[R][R]([H])=Cc1ccccc1</smiles>

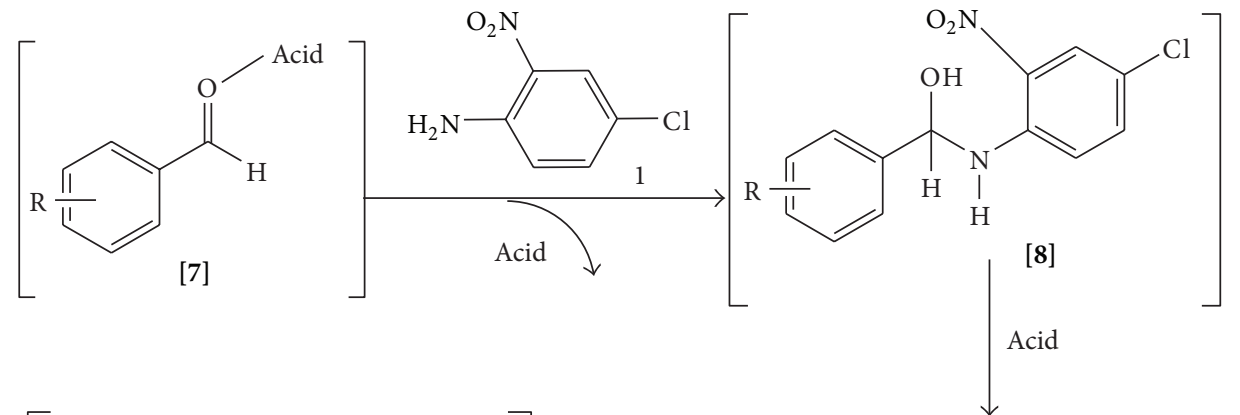

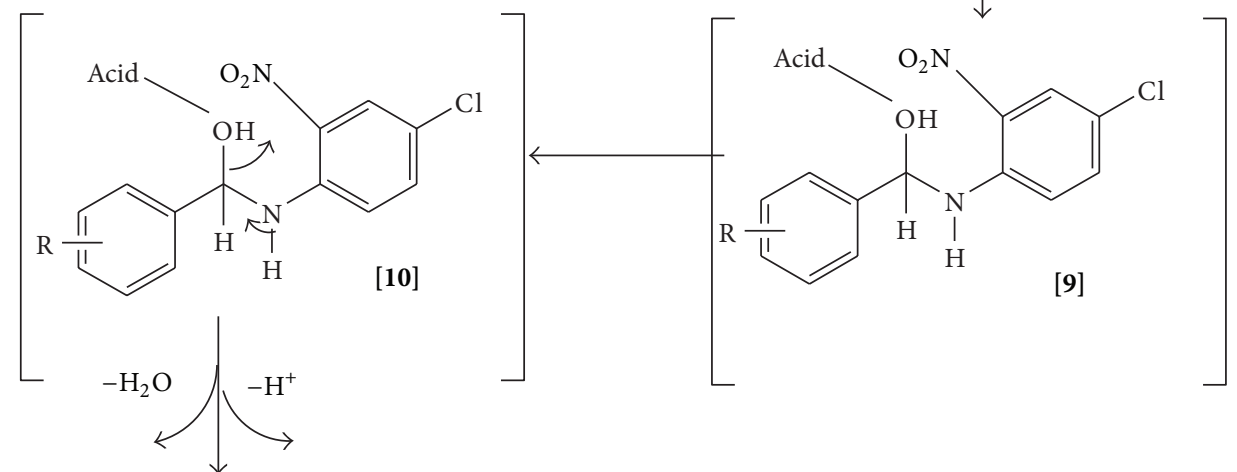<smiles>[R]c1ccc(C(=Nc2ccc(Cl)cc2[N+](=O)[O-])c2ccccc2)cc1</smiles>

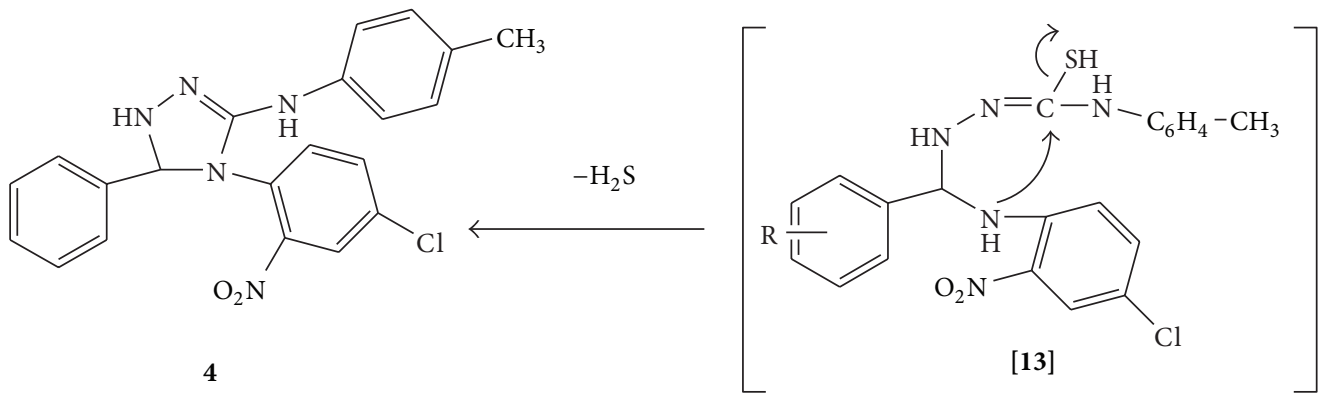

Scheme 2: Plausible mechanism of formation of compound 4. 


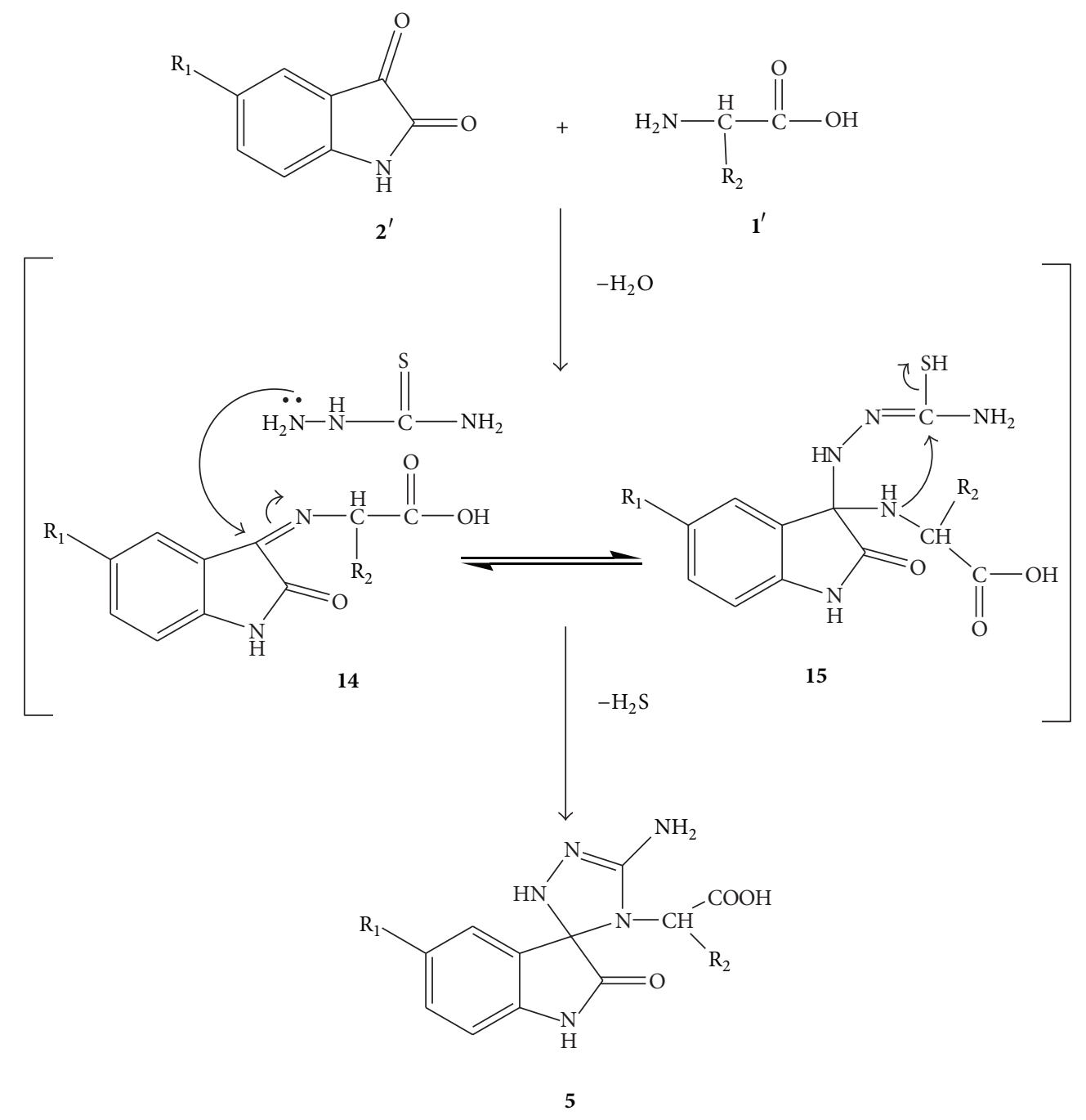

Scheme 3: Plausible mechanism of formation of compound 5.

of $\mathbf{1}^{\prime}, \mathbf{2}^{\prime}$, and 3 in the presence of water containing small amount of alcohol as a solvent. The progress of reaction was monitored by TLC. After completion of reaction, mixture was cooled to room temperature and poured on to crushed ice; the product formed was filtered and recrystallized from ethanol.

Formation of spiro indole-triazoles (5) (reported by us) [31] took place in the absence of natural acid, formation of which is confirmed by spectral analyses.

The third carbonyl group of isatin being more electrophilic forms 3-aryl imino derivatives preferentially [14]. The formation of final compound 5 involves the nucleophilic attack of thiosemicarbazide at the electrophilic indolyl carbon [14] accompanied by the migration of hydrogen atom to form an intermediate thiol derivative [15]. This is followed by cyclization and desulphurization with the loss of hydrogen sulphide to give the suggested spiro compound 5 (Scheme 3 ).

In summary, it can be stated that the present green synthetic protocol is highly efficient as it avoids the use of hazardous solvents at any stage of the reaction. The scope of the method was further studied by reacting differently substituted $1 \mathrm{H}$-indole-2,3diones with amino acids and thiosemicarbazide. The identity of the products (Tables 3 and 4) obtained was confirmed by their IR, ${ }^{1} \mathrm{H} N M R$, and ${ }^{13} \mathrm{C}$ NMR spectral data.

\section{Fluorescence Study}

A fluorescence study was carried out with newly synthesized triazole derivatives $(\mathbf{4 a}-\mathbf{j})$ and $(\mathbf{5 a}-\mathbf{l})$ in order to relate the fluorescence properties to the nature of donating and acceptor groups attached to these moieties. Fluorescence spectra of $5.7 \times 10^{-5} \mathrm{M}$ solutions of compounds were measured; excitation and emission maxima are also reported. Emission spectra of compounds $(\mathbf{4} \mathbf{a}-\mathbf{j})$ and $(\mathbf{5} \mathbf{a}-\mathbf{p})$ were run in DMSO. The resulting triazoles formed by the reaction of $4-\mathrm{Cl}, 2$ $\mathrm{NO}_{2}$ aniline/amino acids and aromatic aldehydes/indole-2,3diones with thiosemicarbazide result in a bathochromic (red) shift of emission maxima (Tables 1 and 2). The combination of 4-chlorophenyl-substituted triazole to 4-chloro-2-nitro aniline in compound $\mathbf{4 d}$ results in the shift of $\lambda \mathbf{e m}$. from 326 to $390.98 \mathrm{~nm}$ owing to the electron's withdrawing nature of $\mathrm{Cl}$ group. Further, combination of imidazole/indole-substituted 
TABLE 1: Fluorescence data of compounds $(\mathbf{4 a - j})$.

\begin{tabular}{lccc}
\hline Entry & $\begin{array}{c}\text { Molar } \\
\text { concentration }\end{array}$ & $\begin{array}{c}\text { 4-Cl, 2- } \mathrm{NO}_{2} \text { aniline } \\
\text { (reactants) } \\
\lambda \text { em·(nm) }\end{array}$ & $\begin{array}{c}\text { Products } \\
\lambda \text { em·(nm) }\end{array}$ \\
\hline $\mathbf{4 a}$ & $5 \times 10^{-5}$ & 326 & 376.969 \\
$\mathbf{4 b}$ & $6 \times 10^{-5}$ & 326 & 362.008 \\
$\mathbf{4 c}$ & $6 \times 10^{-5}$ & 326 & 355.83 \\
$\mathbf{4 d}$ & $5 \times 10^{-5}$ & 326 & 390.98 \\
$\mathbf{4 e}$ & $6 \times 10^{-5}$ & 326 & 354.83 \\
$\mathbf{4 f}$ & $6 \times 10^{-5}$ & 326 & 360.01 \\
$\mathbf{4 g}$ & $6 \times 10^{-5}$ & 326 & 353.63 \\
$\mathbf{4 h}$ & $6 \times 10^{-5}$ & 326 & 356.07 \\
$\mathbf{4 i}$ & $6 \times 10^{-5}$ & 326 & 354.5 \\
$\mathbf{4 j}$ & $6 \times 10^{-5}$ & 326 & 354.26 \\
\hline
\end{tabular}

TABLE 2: Fluorescence data of compounds (5a-1).

\begin{tabular}{lccc}
\hline Entry & $\begin{array}{c}\text { Molar } \\
\text { concentration }\end{array}$ & $\begin{array}{c}1 \mathrm{H} \text {-indole-2,3-diones } \\
\left(\mathrm{H}, 5-\mathrm{Cl}, 5-\mathrm{NO}_{2}\right) \\
\text { reactants } \lambda \text { em.(nm) }\end{array}$ & $\begin{array}{c}\text { Products } \\
\lambda \text { em. }(\mathrm{nm})\end{array}$ \\
\hline $\mathbf{5 a}$ & $7 \times 10^{-5}$ & 296 & 362.008 \\
$\mathbf{5 b}$ & $6 \times 10^{-5}$ & 296 & 360.108 \\
$\mathbf{5 c}$ & $6 \times 10^{-5}$ & 296 & 418.053 \\
$\mathbf{5 e}$ & $6 \times 10^{-5}$ & 296 & 415.915 \\
$\mathbf{5 f}$ & $7 \times 10^{-5}$ & 296 & 386.943 \\
$\mathbf{5 g}$ & $7 \times 10^{-5}$ & 296 & 413.066 \\
$\mathbf{5 h}$ & $6 \times 10^{-5}$ & 296 & 412.116 \\
$\mathbf{5 j}$ & $5 \times 10^{-5}$ & 291 & 422.090 \\
$\mathbf{5 k}$ & $5 \times 10^{-5}$ & 291 & 371.982 \\
$\mathbf{5 l}$ & $5 \times 10^{-5}$ & 291 & 435.864 \\
\hline
\end{tabular}

triazole in $\mathbf{5 j}$ and $\mathbf{5 l}$ to 5 -chloro- $1 H$ indole-2,3-dione also results in red shift. For amino substituents, the bathochromic shift of the fluorescence as well of the absorption band is due to the large electron releasing ability of the nitrogen in amine group (5a-k) (Figures 1, 2, 3, and 4).

\section{Materials and Methods}

4.1. General. Reagents and solvents were obtained from commercial sources and used without further purification. Melting points were determined on a Toshniwal apparatus. The ${ }^{1} \mathrm{H}$ NMR and ${ }^{13} \mathrm{C}$ NMR spectra of synthesized compounds have been carried out at SAIF, Punjab University, Chandigarh. ${ }^{1} \mathrm{H}$ NMR spectra were recorded on Bruker Avance II 400 NMR spectrometer using DMSO- $\mathrm{d}_{6}$ and $\mathrm{CDCl} 3$ as solvent and tetramethylsilane (TMS) as internal reference standard. The purity of compounds was checked on thin layers of silica gel in various nonaqueous solvent systems, for example, benzene : ethyl acetate $(8: 2)$. IR spectra were recorded in $\mathrm{KBr}$ on a Perkin Elmer Infrared L1600300 spectrum Two Li-Ta spectrophotometer and fluorescence studies were carried out on RF-5301 PC spectrofluorophotometer, Shimadzu at FET, MITS, Laxmangarh, Sikar, Rajasthan, India.

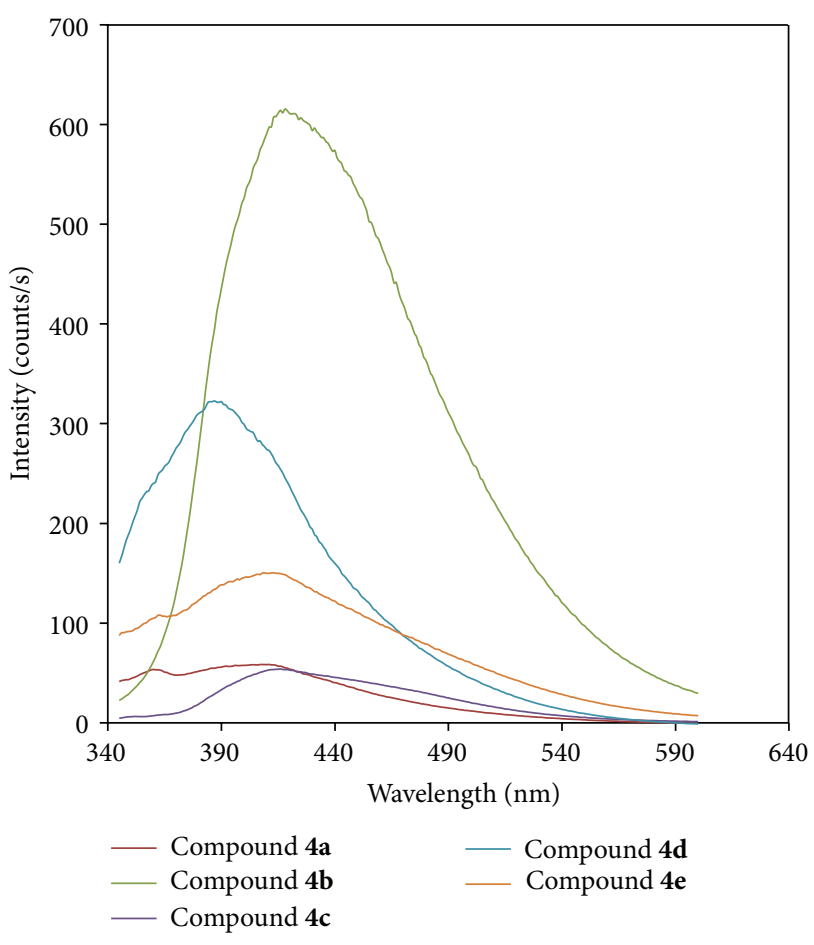

FIGURE 1: Fluorescence spectra of compounds (4a-e).

4.2. General Procedure for the Synthesis of Compounds (4aj). An equimolar mixture of substituted aromatic aldehyde (0.01 mol), 4-chloro-2-nitro aniline $(0.01 \mathrm{~mol})$, and methyl phenyl thiosemicarbazide in water $(15 \mathrm{~mL}$ containing $1-2 \mathrm{~mL}$ of ethanol) in the presence of lemon juice $(2 \mathrm{~mL})$ was mixed in round bottom flask and the mixture was refluxed for the time needed to complete the reaction (as monitored by TLC). After completion of the reaction, mixture was cooled to room temperature and the solid mass was filtered and recrystallized from ethanol.

4.3. General Procedure for the Synthesis of Compounds (5a$p)$. An equimolar mixture of substituted $1 \mathrm{H}$-indole-2,3dione $(0.012 \mathrm{~mol})$, substituted amino acids $(0.012 \mathrm{~mol})$ and thiosemicarbazide in water $(15 \mathrm{~mL}$ containing $1-2 \mathrm{~mL}$ of ethanol) was mixed in round bottom flask and the mixture was refluxed for the time needed to complete the reaction (as monitored by TLC). The initial syrupy reaction mixture solidifies within 2-3 hours. After completion of the reaction, mixture was cooled to room temperature and the solid mass was poured onto crushed ice, filtered, and recrystallized from ethanol.

\section{Antimicrobial Activity}

Synthesized compounds $\mathbf{4} \mathbf{a}-\mathbf{j}$ and $\mathbf{5} \mathbf{a}-\mathbf{p}$ were evaluated for antibacterial and antifungal activity.

5.1. Antibacterial Activity. Synthesized compounds (4a-j) were screened for their antibacterial activity against Grampositive bacteria Bacillus licheniformis, Staphylococcus aureus, 
TABLE 3: Physical characterization data of compounds (4a-j).

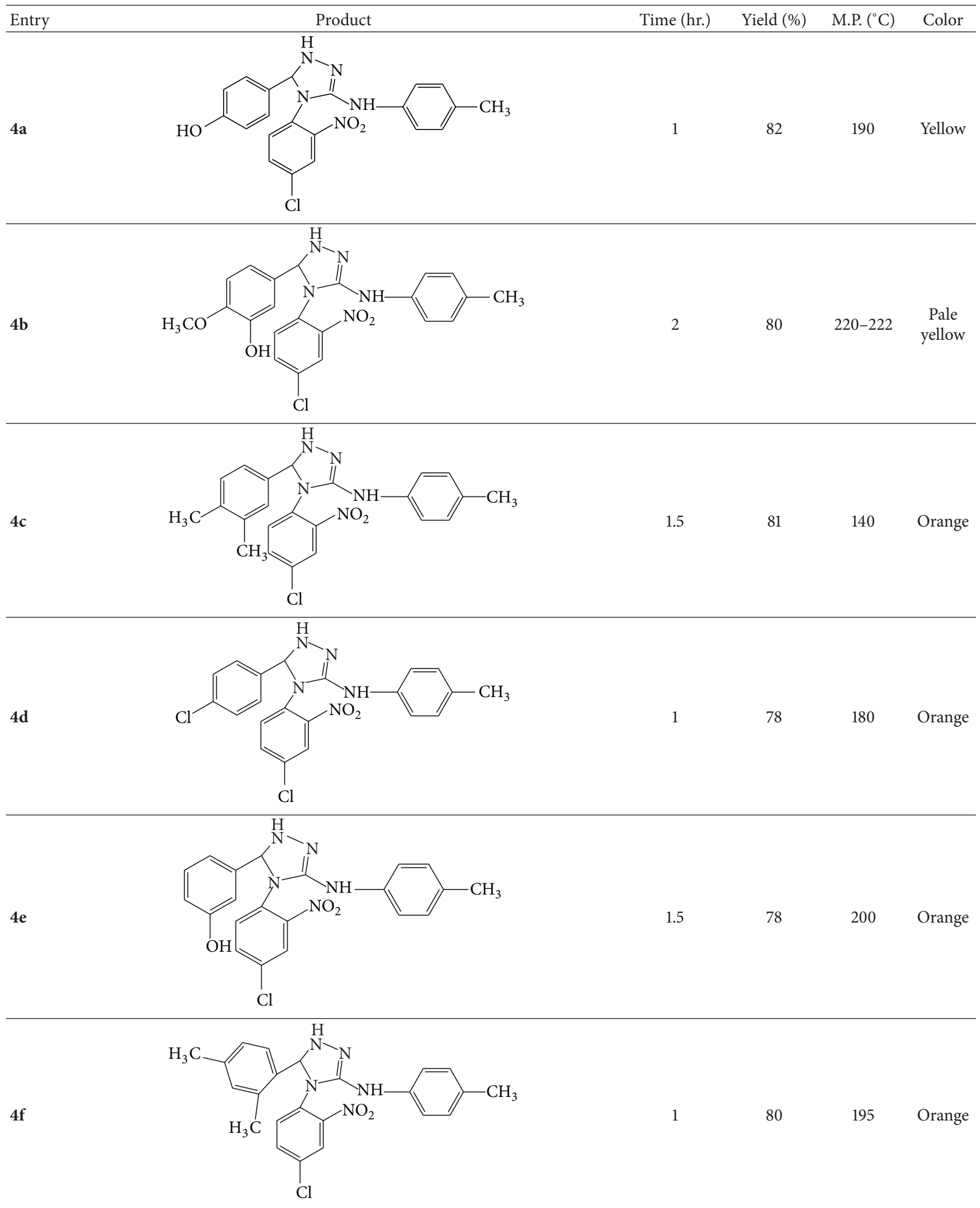


TABLe 3: Continued.

Entry

$4 \mathbf{i}$<smiles>COc1ccc(C2NN=C(Nc3ccc(C)cc3)N2c2ccc(Cl)cc2[N+](=O)[O-])cc1</smiles><smiles>COc1ccc(C2NN=C(Nc3ccc(C)cc3)N2c2ccc(Cl)cc2[N+](=O)[O-])cc1OC</smiles>

and Micrococcus luteus and Gram-negative bacteria Pseudomonas aeruginosa and Escherichia coli by the agar well diffusion method. $5 \mathrm{~mL}$ aliquot of nutrient broth was inoculated with the test organism and incubated at $37^{\circ} \mathrm{C}$ for 24 hours. Sterile nutrient agar plates were also prepared and holes of $5 \mathrm{~mm}$ diameter were cut using a sterile cork borer ensuring proper distribution. The test organisms after 24 hours of incubation were spread onto separate agar plates. The chemical compounds were dissolved in DMSO at a particular concentration or poured into appropriately labelled holes using a pipette in aseptic conditions. A hole containing DMSO served as a control. The plates were left at room temperature for two hours to allow the diffusion of the sample followed by incubation at $37^{\circ} \mathrm{C}$ for 24 hours in inverted position. The antimicrobial activity was determined by measuring the diameter of the zone $(\mathrm{mm})$ showing complete inhibition with respect to control (DMSO) and reference compounds streptomycin and erythromycin. It has been observed that all the compounds tested showed good to excellent activity against tested bacteria.

Compound $\mathbf{4 g}$ shows good activity against bacteria Staphylococcus aureus at $250 \mathrm{ppm}$ concentration. On the other hand, compound $\mathbf{4} \mathbf{j}$ shows excellent activity against bacteria Pseudomonas aeruginosa, Staphylococcus aureus, and Micrococcus luteus at $500 \mathrm{ppm}$ concentration. Compound $\mathbf{4 b}$ shows good activity against bacteria Staphylococcus aureus, Micrococcus luteus, and Escherichia coli and compound 4d also shows good activity against bacteria Pseudomonas aeruginosa, Bacillus licheniformis, and Micrococcus luteus at $500 \mathrm{ppm}$ concentration (Table 5, Figure 5). 
TABle 4: Physical characterization data of compounds (5a-p).

\begin{tabular}{|c|c|c|c|c|c|}
\hline Entry & Product & Time (hr) & Yield (\%) & M.P. $\left({ }^{\circ} \mathrm{C}\right)$ & Color \\
\hline $5 a$ & & 1.5 & 80 & 105 & Red \\
\hline $5 b$ & & 1.5 & 72 & 98 & Brown \\
\hline $5 c$ & & 2 & 76 & 140 & $\begin{array}{c}\text { Dark } \\
\text { brown }\end{array}$ \\
\hline $5 d$ & & 2 & 72 & 185 & $\begin{array}{c}\text { Dark } \\
\text { brown }\end{array}$ \\
\hline $5 e$ & & 1.5 & 77 & 170 & Orange \\
\hline $5 f$ & & 1 & 78 & 195 & Orange \\
\hline
\end{tabular}


TABLE 4: Continued.

\begin{tabular}{|c|c|c|c|c|c|}
\hline Entry & Product & Time (hr) & Yield (\%) & M.P. $\left({ }^{\circ} \mathrm{C}\right)$ & Color \\
\hline $5 g$ & & 1.5 & 75 & 90 & Brown \\
\hline $5 \mathrm{~h}$ & & 1.5 & 75 & $140-142$ & Red \\
\hline $5 i$ & & 1.5 & 71 & 153 & Yellow \\
\hline $5 j$ & & 2 & 70 & $180-182$ & Orange \\
\hline
\end{tabular}

$5 k$<smiles>CSCCC(C(=O)O)N1C(N)=NNC12C(=O)Nc1ccc(Cl)cc12</smiles>

1.5

70

$220 \quad$ Red

51<smiles>NC1=NNC2(C(=O)Nc3ccc(Cl)cc32)N1C(CC1CNc2ccccc21)C(=O)O</smiles><smiles>NC1=NNC2(C(=O)Nc3ccc([N+](=O)[O-])cc32)N1C(Cc1ccccc1)C(=O)O</smiles> 
TABLE 4: Continued.

\begin{tabular}{|c|c|c|c|c|c|}
\hline Entry & Product & Time (hr) & Yield (\%) & M.P. $\left({ }^{\circ} \mathrm{C}\right)$ & Color \\
\hline $5 n$ & & 1.5 & 69 & 207 & $\begin{array}{c}\text { Pale } \\
\text { yellow }\end{array}$ \\
\hline 50 & & 2 & 70 & 194 & $\begin{array}{c}\text { Pale } \\
\text { yellow }\end{array}$ \\
\hline $5 p$ & & 2 & 70 & 185 & $\begin{array}{c}\text { Pale } \\
\text { yellow }\end{array}$ \\
\hline
\end{tabular}

TABLe 5: Antibacterial evaluation of the synthesized compounds (4a-j).

\begin{tabular}{|c|c|c|c|c|c|c|c|c|c|c|}
\hline \multirow{3}{*}{ Compounds } & \multicolumn{10}{|c|}{ Zone of inhibition $(\mathrm{mm})$} \\
\hline & \multicolumn{5}{|c|}{$(250 \mathrm{ppm})$} & \multicolumn{5}{|c|}{ (500 ppm) } \\
\hline & A & B & $\mathrm{C}$ & $\mathrm{D}$ & $\mathrm{E}$ & A & $\mathrm{B}$ & $\mathrm{C}$ & $\mathrm{D}$ & $\mathrm{E}$ \\
\hline $4 a$ & 6 & 8 & 7 & 9 & 9 & 9 & 6 & 10 & 7 & - \\
\hline $4 b$ & 7 & 6 & 9 & 8 & - & 9 & 9 & 11 & 10 & 11 \\
\hline $4 c$ & - & 9 & 9 & - & 8 & 4 & 9 & 4 & - & 9 \\
\hline $4 d$ & 6 & - & - & 8 & - & 10 & 10 & - & 10 & - \\
\hline $4 e$ & 8 & 9 & 6 & 9 & 6 & 9 & 4 & 6 & 10 & 3 \\
\hline $4 f$ & - & - & 9 & 7 & 8 & 9 & - & 9 & 9 & 10 \\
\hline $4 g$ & 7 & 8 & 10 & - & 7 & - & 10 & - & - & 11 \\
\hline $4 h$ & 7 & - & 9 & 9 & - & 3 & - & 10 & 6 & - \\
\hline $4 \mathbf{i}$ & 9 & 7 & - & 8 & 9 & - & 8 & 9 & - & 10 \\
\hline $4 j$ & 9 & 9 & - & 9 & - & 10 & 9 & 11 & 11 & - \\
\hline DMSO & - & - & - & - & - & - & - & - & - & - \\
\hline Streptomycin & 13 & 10 & 14 & 10 & 11 & 10 & 10 & 14 & 14 & 12 \\
\hline Erythromycin & 12 & 11 & - & 12 & - & 12 & 10 & - & 11 & 13 \\
\hline
\end{tabular}

A: Pseudomonas aeruginosa, B: Bacillus licheniformis, C: Staphylococcus aureus, D: Micrococcus luteus, E: Escherichia coli. Bold numbers indicate compounds showing good activity. 


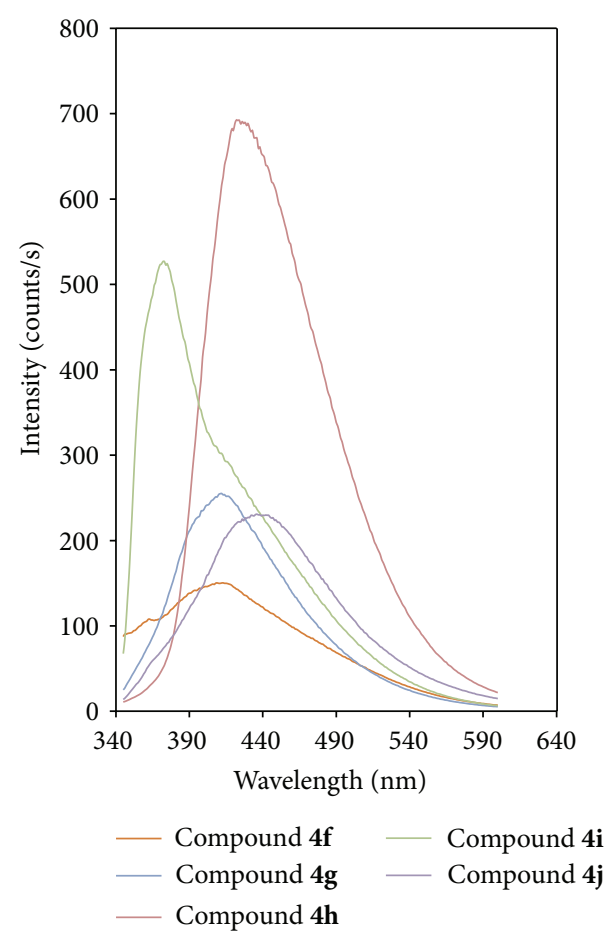

FIGURE 2: Fluorescence spectra of compounds $(\mathbf{4} \mathbf{f}-\mathbf{j})$.

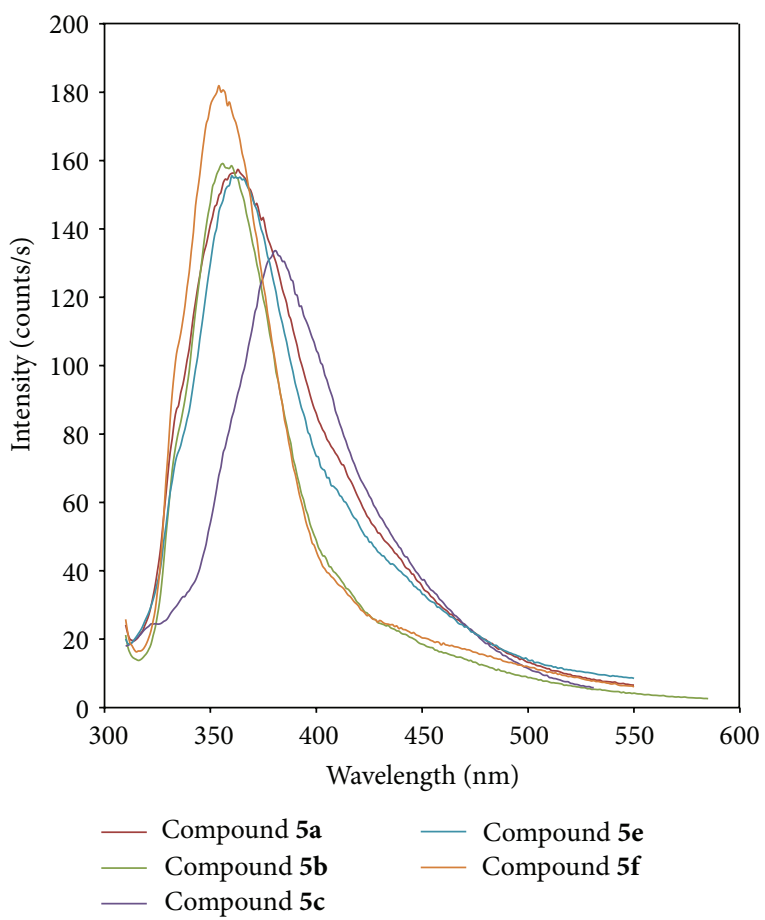

FIgURE 3: Fluorescence spectra of compounds (5a-f).

5.2. Antifungal Activity. Antifungal activity was performed by cup plate method at concentration $500 \mathrm{ppm}$ and $250 \mathrm{ppm}$ against Aspergillus niger, Penicillum sp. fusarium oxysporum, Alternaria brassicicola, Chaetomium orium, and Lycopodium sp. by measuring the zone of inhibition in $\mathrm{mm}$. Sabour and dextrose agar were employed as culture medium, by pouring

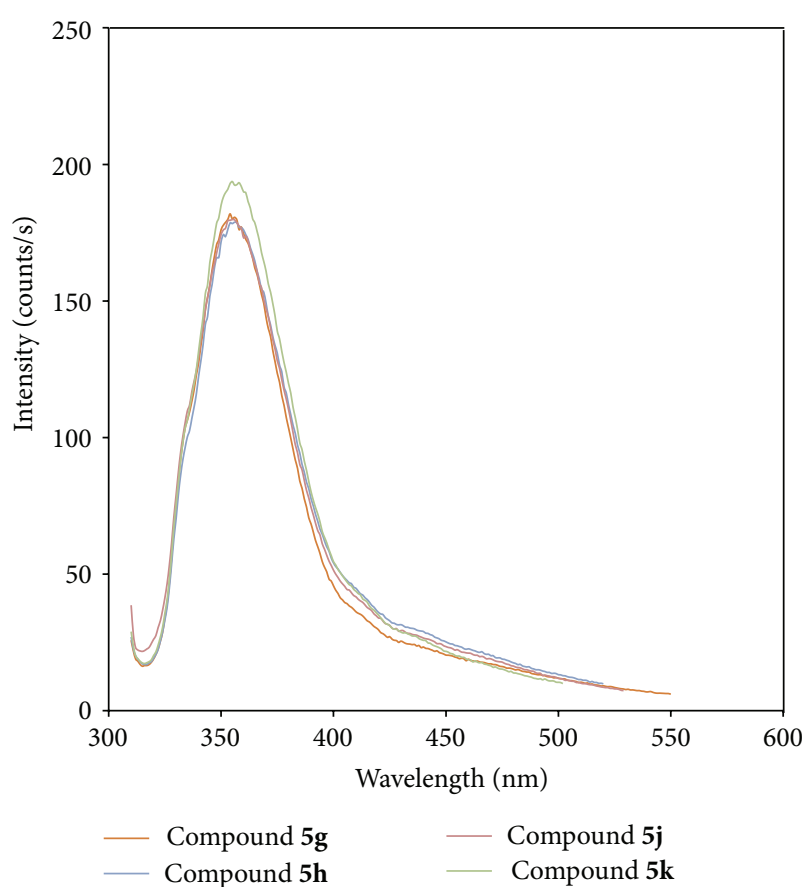

FIGURE 4: Fluorescence spectra of compounds (5g-k).

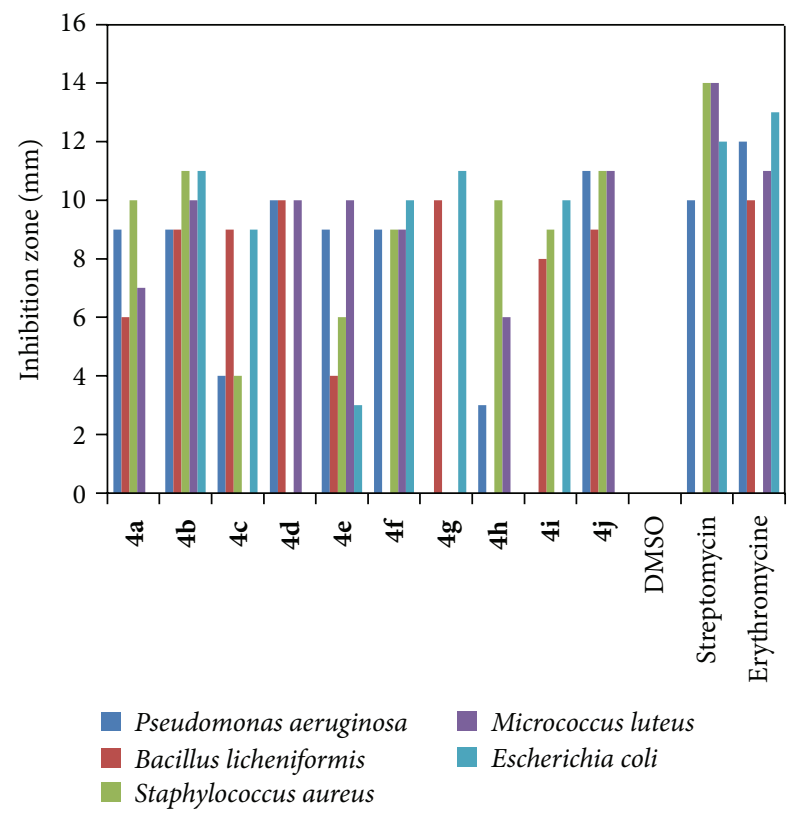

Figure 5: Antibacterial evaluation of the synthesized compounds $(\mathbf{4 a - j})$ at $500 \mathrm{ppm}$.

the sterile agar into Petri dishes in aseptic conditions. $0.1 \mathrm{~mL}$ of each standardized test organism culture was spread onto agar plates. The test compounds (250 and 500 ppm), the standard drug solutions, and the solvent control (DMSO) were placed in the cavity separately. Then the plates were maintained at $-4^{\circ} \mathrm{C}$ for 1 hour to allow the diffusion of solution into the medium. All the fungal plates were incubated at $28^{\circ} \mathrm{C}$ for $42-72 \mathrm{hrs}$ and the zone of inhibition was measured in $\mathrm{mm}$. 
TABLE 6: Antifungal evaluation of the synthesized compounds $(\mathbf{4 a - j})$.

\begin{tabular}{|c|c|c|c|c|c|c|c|c|c|c|c|c|}
\hline \multirow{3}{*}{ Compounds } & \multicolumn{12}{|c|}{ Zone of inhibition (mm) } \\
\hline & \multicolumn{6}{|c|}{ Concentration (250 ppm) } & \multicolumn{6}{|c|}{ Concentration (500 ppm) } \\
\hline & A & $\mathrm{B}$ & $\mathrm{C}$ & $\mathrm{D}$ & $\mathrm{E}$ & $\mathrm{F}$ & A & $\mathrm{B}$ & $\mathrm{C}$ & $\mathrm{D}$ & $\mathrm{E}$ & $\mathrm{F}$ \\
\hline $4 a$ & 7 & 9 & 8 & 8 & 8 & 8 & 8 & 8 & 10 & 10 & 9 & 10 \\
\hline $4 b$ & 6 & 7 & 9 & - & - & - & - & 9 & 9 & 10 & - & 11 \\
\hline $4 c$ & - & 9 & - & 5 & 9 & 5 & - & 8 & 9 & - & 12 & 9 \\
\hline $4 d$ & 5 & - & 10 & - & - & 8 & 9 & - & 9 & - & 11 & - \\
\hline $4 e$ & - & 8 & 8 & - & 8 & - & 7 & - & 11 & - & - & - \\
\hline $4 f$ & 7 & 7 & 9 & 9 & - & 6 & - & 8 & 9 & 11 & - & 12 \\
\hline $4 g$ & 6 & 6 & 10 & 8 & - & - & 9 & 7 & 10 & 9 & 9 & 10 \\
\hline $4 h$ & - & 9 & - & - & 9 & - & 8 & 9 & 11 & - & 11 & 11 \\
\hline $4 i$ & 8 & 8 & - & - & 8 & 8 & - & 6 & - & - & 10 & - \\
\hline $4 j$ & 4 & - & 8 & 8 & - & - & 9 & - & 11 & 8 & 9 & - \\
\hline DMSO & - & - & - & - & - & - & - & - & - & - & - & - \\
\hline Streptomycin & 11 & 11 & 13 & 12 & 13 & 10 & 11 & - & 12 & 13 & 15 & 14 \\
\hline Erythromycin & 14 & 13 & 10 & 11 & 12 & 13 & 14 & 10 & - & 14 & 14 & 12 \\
\hline
\end{tabular}

A: Aspergillus niger, B: Penicillium notatum, C: Fusarium oxysporum, D: Alternaria brassicicola, E: Chaetomium orium, F: Lycopodium sp.

Bold numbers indicate compounds showing good activity.

The antimicrobial activity was determined by measuring the diameter of the zone ( $\mathrm{mm})$ showing complete inhibition with respect to control (DMSO) and reference compounds streptomycin and erythromycin.

Compounds $\mathbf{4 d}$ and $\mathbf{4 g}$ show good activity against fungus Fusarium oxysporum at $250 \mathrm{ppm}$ due to presence of $\mathrm{Cl}$ and $-\mathrm{OH}$ substituents. Compound $4 \mathrm{c}$ shows excellent activity against fungus Chaetomium orium and $\mathbf{4 f}$ shows excellent activity against fungus Alternaria brassicicola and Lycopodium sp. at 500 ppm. Compound 4 h shows excellent activity against fungus Fusarium oxysporum, Chaetomium orium, and Lycopodium sp. at 500 ppm. Compound 4a shows good activity against fungus Fusarium oxyforum, Alternaria brassicicola, and Lycopodium sp. at $500 \mathrm{ppm}$ concentration due to the presence of $-\mathrm{OH}$ substituent (Table 6, Figure 6).

5.3. Antibacterial Activity. Compound 5j shows good activity against bacteria Pseudomonas aeruginosa and 5k shows good activity against bacteria Staphylococcus aureus, respectively at $250 \mathrm{ppm}$ concentration. Compound $\mathbf{5 m}$ shows excellent activity against bacteria Staphylococcus aureus at $250 \mathrm{ppm}$ concentration due to presence of $-\mathrm{NO}_{2}$ substituent. On the other hand, compounds $\mathbf{5 b}, \mathbf{5 g}$, and $\mathbf{5 n}$ show excellent activity against bacteria Escherichia coli at 500 ppm concentration (Table 7, Figure 7).

5.4. Antifungal Activity. Compound 5d shows good activity against Fusarium oxysporum at 250 ppm concentration. Compounds $\mathbf{5 b}, \mathbf{5 h}$, and $\mathbf{5 m}$ show good activity against Lycopodium sp. at $500 \mathrm{ppm}$ concentration. Compounds $\mathbf{5 d}$ and $\mathbf{5 h}$ show good activity against Chaetomium orium at 500 ppm concentration. Compounds $\mathbf{5 f}$ and $\mathbf{5 p}$ also show good activity against Alternaria brassicicola at $500 \mathrm{ppm}$

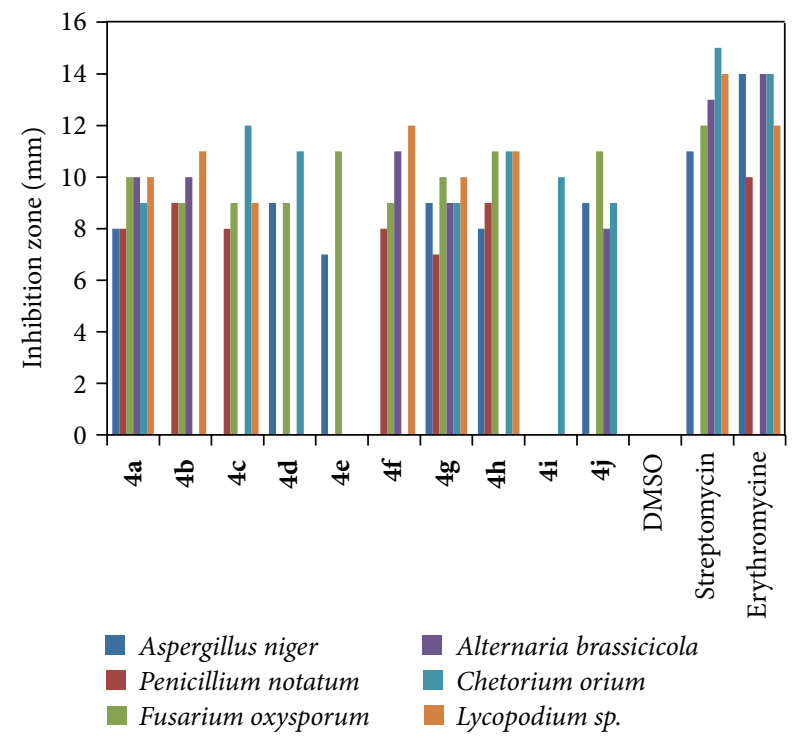

Figure 6: Antifungal evaluation of the synthesized compounds $(\mathbf{4 a - j})$ at $500 \mathrm{ppm}$.

concentration; compounds $\mathbf{5 e}, \mathbf{5 h}$, and $5 \mathbf{j}$ also show good activity against Fusarium oxysporum at 500 ppm concentration (Table 8, Figure 8).

\section{Spectroscopic Characterization Data}

\subsection{Spectroscopic Characterization Data of $\mathbf{4 a}-\mathbf{j}$}

4-(5-(p-Tolylamino)-4-(4-chloro-2-nitrophenyl)-3,4-dihydro2H-1,2,4-triazol-3-yl)phenol (4a). m.p. $190^{\circ}$ C; IR (KBr): 3476, 
TABLE 7: Antibacterial evaluation of the synthesized compounds (5a-p).

\begin{tabular}{|c|c|c|c|c|c|c|c|c|c|c|}
\hline \multirow{3}{*}{ Compounds } & \multicolumn{10}{|c|}{ Zone of inhibition $(\mathrm{mm})$} \\
\hline & \multicolumn{5}{|c|}{$(250 \mathrm{ppm})$} & \multicolumn{5}{|c|}{ (500 ppm) } \\
\hline & $\mathrm{A}$ & $\mathrm{B}$ & $\mathrm{C}$ & $\mathrm{D}$ & $\mathrm{E}$ & $\mathrm{A}$ & B & $\mathrm{C}$ & $\mathrm{D}$ & $\mathrm{E}$ \\
\hline $5 a$ & 8 & 8 & 7 & 9 & 9 & 9 & 6 & 10 & 7 & - \\
\hline $5 b$ & 6 & 6 & 9 & 8 & - & - & 9 & 11 & 10 & 11 \\
\hline $5 c$ & 9 & 9 & 9 & - & 8 & 4 & 9 & 4 & - & 9 \\
\hline $5 d$ & - & - & - & 8 & - & 10 & 10 & - & 10 & 4 \\
\hline $5 e$ & 8 & 9 & 6 & 9 & 6 & - & 4 & 6 & 10 & 3 \\
\hline $5 \mathrm{f}$ & 7 & - & 9 & 7 & 8 & 9 & - & 9 & 9 & 10 \\
\hline $5 g$ & - & 8 & 10 & - & 7 & - & 10 & - & - & 11 \\
\hline $5 \mathrm{~h}$ & 7 & - & 9 & 9 & - & 3 & - & 10 & 6 & 5 \\
\hline $5 \mathbf{i}$ & 9 & 7 & - & 8 & 9 & - & 8 & 9 & - & 10 \\
\hline $5 j$ & 11 & 9 & - & 9 & - & 10 & 9 & 11 & 11 & - \\
\hline $5 k$ & 9 & - & 11 & - & 8 & 8 & 8 & 12 & 2 & 10 \\
\hline 51 & 9 & 7 & - & 9 & 9 & - & 9 & - & 10 & 10 \\
\hline $5 \mathrm{~m}$ & - & - & 12 & 8 & - & 9 & - & 11 & 9 & - \\
\hline $5 n$ & 9 & 8 & 8 & 7 & 9 & - & - & - & 9 & 11 \\
\hline 50 & 10 & 8 & 10 & 9 & 7 & 8 & 8 & 12 & 4 & 9 \\
\hline $5 p$ & 9 & 7 & 9 & 6 & - & 9 & 9 & 11 & 8 & - \\
\hline DMSO & - & - & - & - & - & - & - & - & - & - \\
\hline Streptomycin & 13 & 10 & 14 & 10 & 11 & 10 & - & 14 & 14 & 12 \\
\hline Erythromycin & 12 & 11 & - & 12 & - & 12 & 10 & - & 11 & 13 \\
\hline
\end{tabular}

A: Pseudomonas aeruginosa, B: Bacillus licheniformis, C: Staphylococcus aureus, D: Micrococcus luteus, E: Escherichia coli. Bold numbers indicate compounds showing good activity.

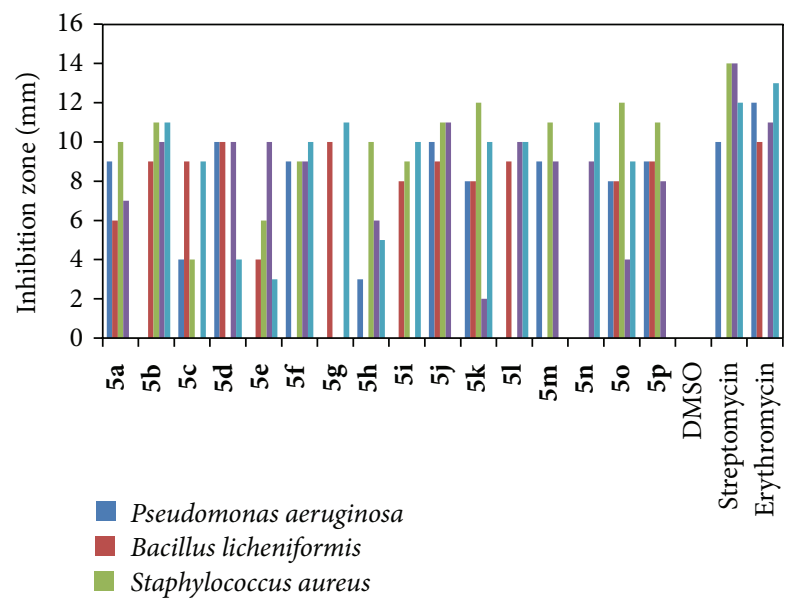

Figure 7: Antibacterial evaluation of the synthesized compounds $5 \mathbf{a}-\mathbf{p}$ at 500 ppm.

$3355,3169,2980,1604,1580,1369,722,699 \mathrm{~cm}^{-1} ;{ }^{1} \mathrm{H} \mathrm{NMR}$ $\left(\delta\right.$ ppm DMSO-d $\left.\mathrm{d}_{6}\right): 2.35\left(\mathrm{~s}, 3 \mathrm{H}, \mathrm{CH}_{3}\right), 5.0(\mathrm{~s}, 1 \mathrm{H}, \mathrm{OH}), 5.04$ (s, $1 \mathrm{H}, \mathrm{CH}), 6.34-7.15(\mathrm{~m}, 4 \mathrm{H}$, aromatic), 6.46-6.95 (m, 4H, aromatic), $6.69-7.25(\mathrm{~m}, 3 \mathrm{H}$, aromatic), $7.01(\mathrm{~s}, 1 \mathrm{H}, \mathrm{N}-\mathrm{H})$, $8.97(\mathrm{~s}, 1 \mathrm{H}, \mathrm{N}-\mathrm{H}) .{ }^{13} \mathrm{C}$ NMR $\left(400 \mathrm{MHz}, \mathrm{DMSO}_{-} \mathrm{d}_{6}\right): 156.92$ $(\mathrm{C}-\mathrm{OH}), 154.98(\mathrm{C}=\mathrm{N}), 141.48-129.72$ (aromatic carbons), 128.08-116.20 (aromatic carbons), 135.82-112.14 (aromatic carbons), $66.92(\mathrm{C}-\mathrm{H}), 24.34\left(\mathrm{CH}_{3}\right) \mathrm{ppm}$. Anal.calcd for $\mathrm{C}_{21} \mathrm{H}_{18} \mathrm{~N}_{5} \mathrm{O}_{3} \mathrm{Cl}$ : C, 59.51; $\mathrm{H}, 4.28$; N, 16.52. Found: C, 59.68; $\mathrm{H}, 4.29 ; \mathrm{N}, 16.53$.

5-(5-(p-Tolylamino)-4-(4-chloro-2-nitrophenyl)-3,4-dihydro2H-1,2,4-triazol-3-yl)-2-methoxyphenol (4b). m.p. 220$222^{\circ} \mathrm{C}$; IR (KBr): $3472,3354,3140,2960,1619,1506,1368$, $1272,1204,1045,701,648 \mathrm{~cm}^{-1} ;{ }^{1} \mathrm{H}$ NMR ( $\delta$ ppm DMSO-d $\left.{ }_{6}\right)$ : $2.38\left(\mathrm{~s}, 3 \mathrm{H}, \mathrm{CH}_{3}\right), 3.73\left(\mathrm{~s}, 3 \mathrm{H}, \mathrm{OCH}_{3}\right), 5.01(\mathrm{~s}, 1 \mathrm{H}, \mathrm{OH})$, $5.06(\mathrm{~s}, 1 \mathrm{H}, \mathrm{CH}), 6.34-6.81(\mathrm{~m}, 4 \mathrm{H}$, aromatic), 6.42-6.51 (m, $3 \mathrm{H}$, aromatic), 6.69-7.98 (m, $3 \mathrm{H}$, aromatic), $7.21(\mathrm{~s}, 1 \mathrm{H}$, $\mathrm{N}-\mathrm{H}), 8.98(\mathrm{~s}, 1 \mathrm{H}, \mathrm{N}-\mathrm{Hs}) .{ }^{13} \mathrm{C}$ NMR $\left(400 \mathrm{MHz}, \mathrm{DMSO}-\mathrm{d}_{6}\right)$ : $156.55(\mathrm{C}-\mathrm{OH}), 154.98(\mathrm{C}=\mathrm{N}), 149.16\left(\mathrm{COCH}_{3}\right), 141.48-$ 135.82 (aromatic carbons), 129.72-123.22 (aromatic carbons), 124.12-112.14 (aromatic carbons), $66.92(\mathrm{C}-\mathrm{H}), \quad 56.20$ $\left(\mathrm{COCH}_{3}\right) 24.32\left(\mathrm{CH}_{3}\right)$ ppm. Anal.calcd for $\mathrm{C}_{22} \mathrm{H}_{20} \mathrm{ClN}_{5} \mathrm{O}_{4}$ : C, 58.22; H, 4.44; N, 15.43. Found: C, 58.05; H, 4.27; N, 15.42.

4-(4-Chloro-2-nitrophenyl)-4,5-dihydro-5-(3,4-dimethylphen$y l$ )-N-p-tolyl-1H-1,2,4-triazol-3-amine (4c). m.p. $140^{\circ} \mathrm{C}$; IR 
TABLE 8: Antifungal evaluation of the synthesized compounds (5a-p).

\begin{tabular}{|c|c|c|c|c|c|c|c|c|c|c|c|c|}
\hline \multirow{3}{*}{ Compounds } & \multicolumn{12}{|c|}{ Zone of inhibition (mm) } \\
\hline & \multicolumn{6}{|c|}{ Concentration (250 ppm) } & \multicolumn{6}{|c|}{ Concentration $(500 \mathrm{ppm})$} \\
\hline & A & B & $\mathrm{C}$ & $\mathrm{D}$ & $\mathrm{E}$ & $\mathrm{F}$ & A & $\mathrm{B}$ & $\mathrm{C}$ & $\mathrm{D}$ & $\mathrm{E}$ & $\mathrm{F}$ \\
\hline $5 a$ & 7 & 9 & 8 & 9 & 8 & 8 & 8 & 8 & 10 & 10 & 9 & 10 \\
\hline $5 \mathbf{b}$ & 8 & 7 & 9 & - & - & - & - & 9 & 9 & 10 & - & 11 \\
\hline $5 c$ & 6 & 9 & - & 8 & 9 & 5 & - & 8 & 9 & - & 12 & 9 \\
\hline $5 d$ & 4 & - & 10 & - & - & 8 & 9 & - & 9 & - & 11 & - \\
\hline $5 e$ & - & 8 & 8 & - & 8 & - & 7 & - & 11 & - & - & - \\
\hline $5 f$ & 7 & 7 & 9 & 9 & - & 6 & - & 8 & 9 & 11 & - & 12 \\
\hline $5 \mathrm{~g}$ & 6 & 6 & 10 & 8 & - & - & 9 & 7 & 10 & 9 & 9 & 10 \\
\hline $5 \mathrm{~h}$ & 8 & 9 & - & - & 9 & - & 8 & 9 & 11 & - & 11 & 11 \\
\hline $5 \mathbf{i}$ & 8 & 8 & - & - & 8 & 8 & - & 6 & - & - & 10 & - \\
\hline $5 \mathbf{j}$ & 4 & - & 8 & 8 & - & - & 9 & - & 11 & 8 & 9 & - \\
\hline $5 \mathrm{k}$ & 7 & - & 9 & 10 & - & - & 8 & 9 & - & 7 & - & - \\
\hline 51 & - & 9 & - & - & 9 & 6 & - & 8 & 10 & 10 & 9 & - \\
\hline $5 \mathrm{~m}$ & 9 & 10 & 9 & 9 & - & 6 & - & - & 9 & 9 & 9 & 11 \\
\hline $5 n$ & - & 9 & 8 & 7 & 8 & 8 & - & - & 9 & - & 9 & 9 \\
\hline 50 & 9 & - & - & - & - & 7 & 8 & - & - & 10 & 7 & 10 \\
\hline $5 p$ & 8 & 8 & 9 & 7 & 7 & 7 & 7 & 9 & 9 & 11 & 12 & 9 \\
\hline DMSO & - & - & - & - & - & - & - & - & - & - & - & - \\
\hline Streptomycin & 11 & 11 & 13 & 12 & 13 & 10 & 11 & - & 12 & 13 & 15 & 14 \\
\hline Erythromycin & 14 & 13 & 10 & 11 & 12 & 13 & 14 & 10 & - & 14 & 14 & 12 \\
\hline
\end{tabular}

A: Aspergillus niger, B: Penicillium notatum, C: Fusarium oxysporum, D: Alternaria brassicicola, E: Chaetomium orium, F: Lycopodium sp. Bold numbers indicate compounds showing good activity.

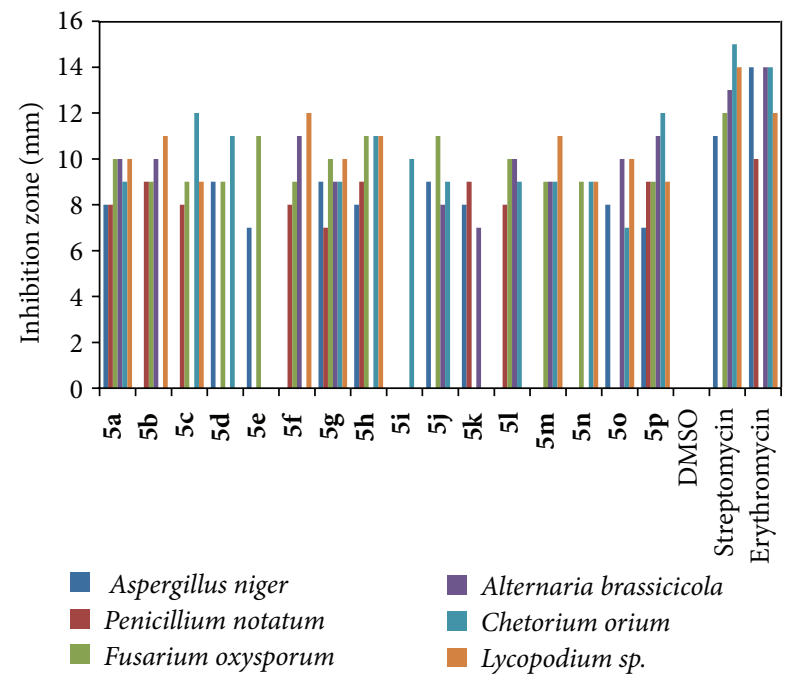

FIGURE 8: Antifungal evaluation of the synthesized compounds $(\mathbf{5 a}-\mathbf{p})$ at $500 \mathrm{ppm}$.

(KBr): 3442, 3317, 3110, 2980, 1645, 1600, 1510, 1350, 1260, $1091,745,660 \mathrm{~cm}^{-1} ;{ }^{1} \mathrm{H}$ NMR $(\delta$ ppm DMSO-d 6 ): $2.35(\mathrm{~s}, 3 \mathrm{H}$, $\left.\mathrm{CH}_{3}\right), 2.35\left(\mathrm{~s}, 3 \mathrm{H}, \mathrm{CH}_{3}\right), 2.35\left(\mathrm{~s}, 3 \mathrm{H}, \mathrm{CH}_{3}\right), 5.10(\mathrm{~s}, 1 \mathrm{H}, \mathrm{CH})$, 6.34-7.18 (m, 4H, aromatic), 6.46-6.95 (m, 3H, aromatic),
6.67-7.20 (m, 3H, aromatic) $7.10(\mathrm{~s}, 1 \mathrm{H}, \mathrm{N}-\mathrm{H}), 9.01(\mathrm{~s}, 1 \mathrm{H}$, $\mathrm{N}-\mathrm{H}) .{ }^{13} \mathrm{C} N \mathrm{MR} \quad\left(400 \mathrm{MHz}, \mathrm{DMSO}_{6}\right): 154.92(\mathrm{C}=\mathrm{N})$, 149.10-135.86 (aromatic carbons), 129.76-116.22 (aromatic carbons), 124.18-112.12 (aromatic carbons), $66.98(\mathrm{C}-\mathrm{H})$, $24.34\left(\mathrm{CH}_{3}\right), 18.12\left(\mathrm{CH}_{3}\right), 17.88\left(\mathrm{CH}_{3}\right) \mathrm{ppm}$. Anal.calcd for $\mathrm{C}_{23} \mathrm{H}_{22} \mathrm{~N}_{5} \mathrm{O}_{2} \mathrm{Cl}$ : C, 63.37; H, 5.09; N, 16.07. Found: C, 63.55; $\mathrm{H}, 5.10 ; \mathrm{N}, 16.09$.

4-(4-Chloro-2-nitrophenyl)-5-(4-chlorophenyl)-4,5-dihydroN-p-tolyl-1H-1,2,4-triazol-3-amine (4d). m.p. $180^{\circ} \mathrm{C}$; IR (KBr): 3440, 3327, 3114, 2981, 1645, 1600, 1511, 1462, 1388, 1291, 1090, $781,642 \mathrm{~cm}^{-1} ;{ }^{1} \mathrm{H}$ NMR $(\delta$ ppm DMSO-d 6$): 2.35(\mathrm{~s}, 3 \mathrm{H}$, $\left.\mathrm{CH}_{3}\right), 5.06(\mathrm{~s}, 1 \mathrm{H}, \mathrm{CH}), 6.34-7.15$ ( $\mathrm{m}, 4 \mathrm{H}$, aromatic), 6.46$6.95(\mathrm{~m}, 4 \mathrm{H}$, aromatic), 6.67-7.20 (m, 3H, aromatic) 7.01 (s, 1H, N-H), 8.83 (s, 1H, N-H). ${ }^{13} \mathrm{CNMR}(400 \mathrm{MHz}$, DMSO- $\left.\mathrm{d}_{6}\right)$ : $154.94(\mathrm{C}=\mathrm{N}), 149.10-135.88$ (aromatic carbons), 129.78-123.22 (aromatic carbons), 116.28-112.18 (aromatic carbons), $66.92(\mathrm{C}-\mathrm{H}), 24.38\left(\mathrm{CH}_{3}\right), \mathrm{ppm}$. Anal.calcd for $\mathrm{C}_{21} \mathrm{H}_{17} \mathrm{~N}_{5} \mathrm{O}_{2} \mathrm{Cl}_{2}$ : C, 57.03; H, 3.87; N, 15.83. Found: C, 57.21; $\mathrm{H}, 3.86 ; \mathrm{N}, 15.84$.

3-(5-(p-Tolylamino)-4-(4-chloro-2-nitrophenyl)-3,4-dihydro2H-1,2,4-triazol-3-yl)phenol (4e). m.p. $200^{\circ} \mathrm{C}$; IR (KBr): 3476, 3355, 3169, 2980, 1604, 1580, 1369, 722, $699 \mathrm{~cm}^{-1} ;{ }^{1} \mathrm{H} \mathrm{NMR}$ $(\delta$ ppm DMSO-d 6$): 2.35\left(\mathrm{~s}, 3 \mathrm{H}, \mathrm{CH}_{3}\right), 5.0(\mathrm{~s}, 1 \mathrm{H}, \mathrm{OH}), 5.04$ (s, $1 \mathrm{H}, \mathrm{CH}), 6.34-7.15$ (m, $4 \mathrm{H}$, aromatic), 6.46-6.95 (m, $4 \mathrm{H}$, 
aromatic), 6.67-7.20 (m, 3H, aromatic), $7.23(\mathrm{~s}, 1 \mathrm{H}, \mathrm{N}-\mathrm{H})$, 9.03 (s, 1H, N-H). ${ }^{13} \mathrm{C} \mathrm{NMR}\left(400 \mathrm{MHz}, \mathrm{DMSO}-\mathrm{d}_{6}\right): 156.24$ $(\mathrm{C}-\mathrm{OH}), 154.94(\mathrm{C}=\mathrm{N}), 145.10-135.86$ (aromatic carbons), 129.72-123.28 (aromatic carbons), 124.18-112.12 (aromatic carbons), $66.94(\mathrm{C}-\mathrm{H}), 24.32\left(\mathrm{CH}_{3}\right), \mathrm{ppm}$. Anal.calcd for $\mathrm{C}_{21} \mathrm{H}_{18} \mathrm{ClN}_{5} \mathrm{O}_{3}$ : C, 59.51; $\mathrm{H}, 4.28 ; \mathrm{N}, 16.52$. Found: C, 59.34; $\mathrm{H}, 4.27$; N, 16.51 .

4-(4-Chloro-2-nitrophenyl)-4,5-dihydro-5-(2,4-dimethylphenyl)-N-p-tolyl-1H-1,2,4-triazol-3-amine (4f). m.p. $195^{\circ} \mathrm{C}$; IR (KBr): 3477, 3330, 3169 2980, 1604, 1600, 1508, 1369, 1091, 722, $699 \mathrm{~cm}^{-1} ;{ }^{1} \mathrm{H}$ NMR $\left(\delta\right.$ ppm DMSO-d 6 ): $2.35\left(\mathrm{~s}, 3 \mathrm{H}, \mathrm{CH}_{3}\right)$, $2.38\left(\mathrm{~s}, 3 \mathrm{H}, \mathrm{CH}_{3}\right), 2.32\left(\mathrm{~s}, 3 \mathrm{H}, \mathrm{CH}_{3}\right), 5.08(\mathrm{~s}, 1 \mathrm{H}, \mathrm{CH}), 6.34-$ 7.15 (m, 4H, aromatic), 6.46-6.95 (m, 3H, aromatic), 6.67-7.20 (m, 3H, aromatic), 7.67 (s, $1 \mathrm{H}, \mathrm{N}-\mathrm{H}), 8.99$ (s, $1 \mathrm{H}, \mathrm{N}-\mathrm{H})$. ${ }^{13} \mathrm{C}$ NMR (400 MHz, DMSO- $\left.\mathrm{d}_{6}\right)$ : $154.96(\mathrm{C}=\mathrm{N}), 140.42-$ 129.74 (aromatic carbons), 135.82-123.22 (aromatic carbons), 124.16-112.16 (aromatic carbons), $66.92(\mathrm{C}-\mathrm{H}), 24.36\left(\mathrm{CH}_{3}\right)$, $24.14\left(\mathrm{CH}_{3}\right), 16.14\left(\mathrm{CH}_{3}\right)$. ppm Anal.calcd for $\mathrm{C}_{23} \mathrm{H}_{22} \mathrm{~N}_{5} \mathrm{O}_{2} \mathrm{Cl}$ : C, 63.37; H, 5.09; N, 16.07. Found: C, 63.54; H, 5.08; N, 16.06.

2-(5-(p-Tolylamino)-4-(4-chloro-2-nitrophenyl)-3,4-dihydro2H-1,2,4-triazol-3-yl)phenol (4g). m.p. $220^{\circ} \mathrm{C}$; IR (KBr): 3476, $3354,3158,1630,1504,1341,1203,723,699 \mathrm{~cm}^{-1} ;{ }^{1} \mathrm{H}$ NMR $(\delta$ ppm DMSO-d 6 ): 2.35 (s, 3H, $\left.\mathrm{CH}_{3}\right), 5.01$ (s, $\left.1 \mathrm{H}, \mathrm{OH}\right), 5.04$ (s, $1 \mathrm{H}, \mathrm{CH}), 6.34-7.15$ (m, 4H, aromatic), 6.46-6.95 (m, 4H, aromatic), 6.67-7.20 (m, 3H, aromatic), $7.0(\mathrm{~s}, 1 \mathrm{H}, \mathrm{N}-\mathrm{H})$, 8.93 (s, 1H, N-H). ${ }^{13} \mathrm{CNMR}\left(400 \mathrm{MHz}, \mathrm{DMSO}_{6}\right.$ ): 156.24 (C-OH), $154.92(\mathrm{C}=\mathrm{N}), 141.42-135.86$ (aromatic carbons), 136.32-124.12 (aromatic carbons), 128.02-112.16 (aromatic carbons), $66.94(\mathrm{C}-\mathrm{H}), 24.32\left(\mathrm{CH}_{3}\right), \mathrm{ppm}$ Anal.calcd for $\mathrm{C}_{21} \mathrm{H}_{18} \mathrm{~N}_{5} \mathrm{O}_{3} \mathrm{Cl}$ : C, 59.51; H, 4.28; N, 16.52. Found: C, 59.33; $\mathrm{H}, 4.26 ; \mathrm{N}, 16.50$.

4-(4-Chloro-2-nitrophenyl)-4,5-dihydro-5-(3-methoxyphenyl)N-p-tolyl-1H-1,2,4-triazol-3-amine (4h). m.p. $210^{\circ} \mathrm{C}$; IR ( $\left.\mathrm{KBr}\right)$ : $3476,3356,2924,1629,1562,1505,1369,1341,1251,1203$, $1158,723,700 \mathrm{~cm}^{-1}$; ${ }^{1} \mathrm{HNMR}\left(\delta \mathrm{ppm} \mathrm{DMSO}-\mathrm{d}_{6}\right): 2.35(\mathrm{~s}$, $\left.3 \mathrm{H}, \mathrm{CH}_{3}\right), 3.73\left(\mathrm{~s}, 3 \mathrm{H}, \mathrm{OCH}_{3}\right), 5.04(\mathrm{~s}, 1 \mathrm{H}, \mathrm{CH}), 6.34-7.15$ (m, $4 \mathrm{H}$, aromatic), 6.46-6.95 $(\mathrm{m}, 4 \mathrm{H}$, aromatic), 6.67-7.98 (m, 3H, aromatic), 7.09 (s, 1H, N-H), 9.01 (s, 1H, N-H). ${ }^{13} \mathrm{CNMR} \quad(400 \mathrm{MHz}, \quad$ DMSO-d 6$): 154.42(\mathrm{C}=\mathrm{N}), 149.16$ $\left(\mathrm{COCH}_{3}\right), 145.62-136.32$ (aromatic carbons), 137.72-128.08 (aromatic carbons), 124.16-112.12 (aromatic carbons), 66.94 (C-H), $56.24\left(\mathrm{COCH}_{3}\right), 24.34\left(\mathrm{CH}_{3}\right), \mathrm{ppm}$, Anal.calcd for $\mathrm{C}_{22} \mathrm{H}_{20} \mathrm{~N}_{5} \mathrm{O}_{3} \mathrm{Cl}$ : C, 60.34; H, 4.60; N, 15.99. Found: C, 60.16; $\mathrm{H}, 4.62$; N, 15.97 .

4-(4-Chloro-2-nitrophenyl)-4,5-dihydro-5-(4-methoxyphenyl)$\mathrm{N}$-p-tolyl-1H-1,2,4-triazol-3 amine (4i). m.p. $240^{\circ} \mathrm{C}$; IR ( $\left.\mathrm{KBr}\right)$ : $3477,3352,2934,1627,1564,1507,1364,1345,1254,1201$, 1150, 727, $700 \mathrm{~cm}^{-1} ;{ }^{1} \mathrm{HNMR}(\delta$ ppm DMSO-d 6$): 2.35(\mathrm{~s}$, $\left.3 \mathrm{H}, \mathrm{CH}_{3}\right), 3.73\left(\mathrm{~s}, 3 \mathrm{H}, \mathrm{OCH}_{3}\right), 5.04(\mathrm{~s}, 1 \mathrm{H}, \mathrm{CH}), 6.34-7.15$ (m, 4H, aromatic), 6.63-6.95 (m, 4H, aromatic), 6.67-7.98 (m, 3H, aromatic), $7.0(\mathrm{~s}, 1 \mathrm{H}, \mathrm{N}-\mathrm{H}), 8.93(\mathrm{~s}, 1 \mathrm{H}, \mathrm{N}-\mathrm{H})$. ${ }^{13} \mathrm{C}$ NMR $\left(400 \mathrm{MHz}, \mathrm{DMSO}-\mathrm{d}_{6}\right): 156.24\left(\mathrm{O}-\mathrm{CH}_{3}\right), 154.44$ $\left(\mathrm{CH}_{3}\right), 150.14\left(\mathrm{COCH}_{3}\right), 145.64-135.82$ (aromatic carbons), 136.34-124.12 (aromatic carbons), 128.02-112.18 (aromatic carbons), $66.98(\mathrm{C}-\mathrm{H}), 55.90\left(\mathrm{COCH}_{3}\right), 55.22\left(\mathrm{CH}_{3}\right), 24.32$
$\left(\mathrm{CH}_{3}\right)$ ppm, Anal.calcd for $\mathrm{C}_{22} \mathrm{H}_{20} \mathrm{~N}_{5} \mathrm{O}_{3} \mathrm{Cl}$ : C, 60.34; H, 4.60; N, 15.98. Found: C, 60.17; H, 4.62; N, 15.96 .

4-(4-Chloro-2-nitrophenyl)-4,5-dihydro-5-(3,4-dimethoxyphenyl)-N-p-tolyl-1H-1,2,4 triazol-3-amine (4j). m.p. $160^{\circ} \mathrm{C}$; IR (KBr): 3476, 3354, 2922, 1627, 1508, 1464, 1339, 1203, 1134, $1044793,699 \mathrm{~cm}^{-1}$; ${ }^{1} \mathrm{H}$ NMR ( $\delta$ ppm DMSO-d 6 ): 2.35 (s, 3H, $\left.\mathrm{CH}_{3}\right), 3.73\left(\mathrm{~s}, 3 \mathrm{H}, \mathrm{OCH}_{3}\right), 3.73\left(\mathrm{~s}, 3 \mathrm{H}, \mathrm{OCH}_{3}\right)$, $5.04(\mathrm{~s}, 1 \mathrm{H}, \mathrm{CH}), 6.34-7.81(\mathrm{~m}, 4 \mathrm{H}$, aromatic), 6.46-6.95 (m, 3H, aromatic), 6.67-7.98 (m, 3H, aromatic), $7.0(\mathrm{~s}, 1 \mathrm{H}$, $\mathrm{N}-\mathrm{H}), 8.93$ (s, 1H, N-H). ${ }^{13} \mathrm{C} \mathrm{NMR}\left(400 \mathrm{MHz}, \mathrm{DMSO}-\mathrm{d}_{6}\right)$ : $154.96(\mathrm{C}=\mathrm{N}), 150.14\left(2 \times \mathrm{COCH}_{3}\right), 149.54\left(\mathrm{O}-\mathrm{CH}_{3}\right), 147.62$ $\left(\mathrm{O}-\mathrm{CH}_{3}\right), 141.48-129.74$ (aromatic carbons), 124.18-120.08 (aromatic carbons), 123.28-112.14 (aromatic carbons), 66.92 $(\mathrm{C}-\mathrm{H}), 56.24\left(\mathrm{CH}_{3}\right), 56.20\left(2 \times \mathrm{COCH}_{3}\right), 24.34\left(\mathrm{CH}_{3}\right) \mathrm{ppm}$ Anal.calcd for $\mathrm{C}_{23} \mathrm{H}_{22} \mathrm{~N}_{5} \mathrm{O}_{4} \mathrm{Cl}$ : C, $59.04 ; \mathrm{H}, 4.74 ; \mathrm{N}, 14.97$. Found: C, 59.22; H, 4.76; N, 14.95 .

\subsection{Spectroscopic Characterization Data of $5 a-p$}

2-(5-Amino-2-oxo-1, 2-dihydrospiro (indole-3, 3-(1, 2, 4) triazol)-4(2H)-yl) propanoic acid (5a). m.p. $105^{\circ} \mathrm{C}$; IR (KBr): 3419, 3159, 2980,1700, 1674, 1593,1465,1060,1091 $\mathrm{cm}^{-1}$; ${ }^{1} \mathrm{H}$ NMR $\left(\delta\right.$ ppm DMSO-d 6 ): $1.23\left(\mathrm{~d}, 3 \mathrm{H}, \mathrm{CH}_{3}\right), 2.00(\mathrm{~s}$, $2 \mathrm{H}, \mathrm{NH}_{2}$ of triazole ring), $3.67(\mathrm{q}, 1 \mathrm{H}, \mathrm{CH}), 7.0(\mathrm{~s}, 1 \mathrm{H}, \mathrm{N}-$ $\mathrm{H}$ of triazole ring), $6.88-7.52(\mathrm{~m}, 4 \mathrm{H}$, aromatic), $8.0(\mathrm{~s}, 1 \mathrm{H}$, $\mathrm{NH}$ of indole ring), $11.0(\mathrm{~s}, 1 \mathrm{H}, \mathrm{OH}) .{ }^{13} \mathrm{C} \mathrm{NMR}(400 \mathrm{MHz}$, DMSO- $\left.\mathrm{d}_{6}\right)$ : 174.12 ( $\mathrm{C}=\mathrm{O}$, acid), 168.24 ( $\mathrm{C}=\mathrm{O}$, indole), 162.14 $(\mathrm{C}=\mathrm{N}), 141.16-130.74$ (aromatic carbons), $76.56(\mathrm{~N}-\mathrm{C}-\mathrm{NH})$, $46.22(\mathrm{CH}), 14.64\left(\mathrm{CH}_{3}\right)$ ppm. Anal.calcd for $\mathrm{C}_{12} \mathrm{H}_{13} \mathrm{~N}_{5} \mathrm{O}_{3}$ : C, 52.36; H, 4.76; N, 25.44. Found: C, 52.53; H, 4.79; N, 25.48.

2-(5-Amino-2-oxo-1, 2-dihydrospiro (indole-3, 3-(1, 2, 4) triazol)-4(2H)-yl)-4-(methylsulfanyl) butanoic acid (5b). m.p. $98^{\circ} \mathrm{C}$; IR (KBr): 3420, 3150, 2982, 1702, 1675, 1590, 1460, 1062, 780, 672 $\mathrm{cm}^{-1} ;{ }^{1} \mathrm{H}$ NMR $(\delta$ ppm DMSO-d 6$): 2.00(\mathrm{~s}$, $2 \mathrm{H}, \mathrm{NH}_{2}$ of triazole ring), $2.01\left(\mathrm{q}, 2 \mathrm{H}, \mathrm{CH}_{2}\right), 2.09(\mathrm{~s}, 3 \mathrm{H}$, $\mathrm{CH}_{3}$ ), 2.44 (t, 2H, $\left.\mathrm{CH}_{2}\right), 3.49$ (t, 1H, CH), $7.1(\mathrm{~s}, 1 \mathrm{H}, \mathrm{N}-\mathrm{H}$ of triazole ring), $6.86-7.54(\mathrm{~m}, 4 \mathrm{H}$, aromatic), 8.1 (s, $1 \mathrm{H}, \mathrm{NH}$ of indole ring), $11.1(\mathrm{~s}, 1 \mathrm{H}, \mathrm{OH}) .{ }^{13} \mathrm{CNMR}(400 \mathrm{MHz}, \mathrm{DMSO}-$ $\left.\mathrm{d}_{6}\right): 174.18(\mathrm{C}=\mathrm{O}$, acid $), 168.26(\mathrm{C}=\mathrm{O}$, indole $), 162.12(\mathrm{C}=\mathrm{N})$, 142.14-122.14 (aromatic carbons), 75.54 (N-C-NH), 51.22 $(\mathrm{CH}), 30.08\left(\mathrm{CH}_{2}\right), 28.14\left(\mathrm{CH}_{2}\right), 17.64\left(\mathrm{CH}_{3}\right)$ ppm. Anal.calcd for $\mathrm{C}_{14} \mathrm{H}_{17} \mathrm{~N}_{5} \mathrm{O}_{3} \mathrm{~S}$ : C, 50.14; $\mathrm{H}, 5.11 ; \mathrm{N}, 20.88$. Found: C, 50.36; $\mathrm{H}, 5.14 ; \mathrm{N}, 20.84$.

2-(5-Amino-2-oxo-1, 2-dihydrospiro (indole-3, 3-(1, 2, 4)triazol)-4(2H)-yl)-3-(1H-imidazole-4-yl)propanoic acid (5c). m.p. $140^{\circ} \mathrm{C}$; IR (KBr): 3422, 3152, 2982, 1708, 1675, 1593, 1530, 1420, $1091 \mathrm{~cm}^{-1} ;{ }^{1} \mathrm{H}$ NMR $\left(\delta\right.$ ppm DMSO-d 6 ): $2.01\left(\mathrm{~s}, 2 \mathrm{H}, \mathrm{NH}_{2}\right.$ of triazole ring), $2.78(\mathrm{dd}, 1 \mathrm{H}, \mathrm{CH}), 3.88(\mathrm{t}, 1 \mathrm{H}, \mathrm{CH}), 6.80$ (s, $1 \mathrm{H}, \mathrm{CH}), 7.0$ (s, 1H, N-H of triazole ring), 7.44 (s, $1 \mathrm{H}$, $\mathrm{CH}), 6.87-7.53(\mathrm{~m}, 4 \mathrm{H}$, aromatic), 8.2 (s, $1 \mathrm{H}, \mathrm{NH}$ of indole ring), 11.0 (s, $1 \mathrm{H}, \mathrm{OH}), 13.4(\mathrm{~s}, 1 \mathrm{H}, \mathrm{NH}) .{ }^{13} \mathrm{C} \mathrm{NMR}(400 \mathrm{MHz}$, DMSO- $\left.\mathrm{d}_{6}\right): 174.18(\mathrm{C}=\mathrm{O}$, acid), 168.26 (C=O, indole), 162.12 $(\mathrm{C}=\mathrm{N}), 142.14-119.62$ (aromatic carbons), $75.54(\mathrm{~N}-\mathrm{C}-\mathrm{NH})$, 
45.22 (CH), $27.12\left(\mathrm{CH}_{2}\right)$ ppm. Anal.calcd for $\mathrm{C}_{15} \mathrm{H}_{15} \mathrm{~N}_{7} \mathrm{O}_{3}: \mathrm{C}$, 52.78; H, 4.43; N, 28.73. Found: C, 52.58; H, 4.45; N, 28.76.

2-(5-Amino-2-oxo-1, 2-dihydrospiro (indole-3, 3-(1, 2, 4)triazol)-4(2H)-yl)-3-(1H-indole-3-yl)propanoic acid (5d). m.p. $185^{\circ} \mathrm{C}$; IR (KBr): 3425, 3155, 2984, 1708, 1677, 1597, 1530, 1420, $1094 \mathrm{~cm}^{-1} ;{ }^{1} \mathrm{H}$ NMR $(\delta$ ppm DMSO-d 6 ): $2.02(\mathrm{~s}, 2 \mathrm{H}$, $\mathrm{NH}_{2}$ of triazole ring), $2.65\left(\mathrm{dd}, 2 \mathrm{H}, \mathrm{CH}_{2}\right), 3.88(\mathrm{t}, 1 \mathrm{H}, \mathrm{CH})$, $7.1(\mathrm{~s}, 1 \mathrm{H}, \mathrm{N}-\mathrm{H}$ of triazole ring), $6.80(\mathrm{~s}, 1 \mathrm{H}, \mathrm{CH}), 6.88-$ 7.52 (m, 4H, aromatic), 7.18-7.20 (m, 4H, aromatic), 8.3 (s, $1 \mathrm{H}, \mathrm{NH}$ of indole ring), $10.1(\mathrm{~s}, 1 \mathrm{H}, \mathrm{NH}), 11.1(\mathrm{~s}, 1 \mathrm{H}$, $\mathrm{OH}) .{ }^{13} \mathrm{CNMR}\left(400 \mathrm{MHz}, \mathrm{DMSO}-\mathrm{d}_{6}\right): 174.18$ (C=O, acid), $168.26(\mathrm{C}=\mathrm{O}$, indole), $162.12(\mathrm{C}=\mathrm{N}), 142.14-110.92$ (aromatic carbons), $75.54(\mathrm{~N}-\mathrm{C}-\mathrm{NH}), 46.22(\mathrm{CH}), 28.12\left(\mathrm{CH}_{2}\right) \mathrm{ppm}$. Anal.calcd for $\mathrm{C}_{20} \mathrm{H}_{18} \mathrm{~N}_{6} \mathrm{O}_{3}$ : C, 61.63; $\mathrm{H}, 4.65 ; \mathrm{N}, 21.53$. Found: C, 61.43; H, 4.68; N, 21.55.

2-(5-Amino-2-oxo-1, 2-dihydrospiro (indole-3, 3-(1, 2, 4) triazol)-4(2H)-yl)-3-methylpentanoic acid (5e). m.p. $170^{\circ} \mathrm{C}$; IR (KBr): 3427, 3150, 2980, 1701, 1672, 1592, 1533, 1424, $1094 \mathrm{~cm}^{-1}$; ${ }^{1} \mathrm{HNMR}\left(\delta\right.$ ppm DMSO-d 6 ): $0.96\left(\mathrm{t}, 3 \mathrm{H}, \mathrm{CH}_{3}\right), 1.06(\mathrm{~d}$, $\left.3 \mathrm{H}, \mathrm{CH}_{3}\right), 1.29\left(\mathrm{~m}, 2 \mathrm{H}, \mathrm{CH}_{2}\right), 2.03\left(\mathrm{~s}, 2 \mathrm{H}, \mathrm{NH}_{2}\right.$ of triazole ring), $2.21\left(\mathrm{~m}, 2 \mathrm{H}, \mathrm{CH}_{2}\right), 3.48(\mathrm{~d}, 1 \mathrm{H}, \mathrm{CH}), 7.0(\mathrm{~s}, 1 \mathrm{H}, \mathrm{N}-$ $\mathrm{H}$ of triazole ring), $6.88-7.52(\mathrm{~m}, 4 \mathrm{H}$, aromatic), $8.1(\mathrm{~s}, 1 \mathrm{H}$, $\mathrm{NH}$ of indole ring), $11.1(\mathrm{~s}, 1 \mathrm{H}, \mathrm{OH}) .{ }^{13} \mathrm{C} \mathrm{NMR}(400 \mathrm{MHz}$, DMSO- $\left.\mathrm{d}_{6}\right): 174.18(\mathrm{C}=\mathrm{O}$, acid), $168.26(\mathrm{C}=\mathrm{O}$, indole), 162.12 $(\mathrm{C}=\mathrm{N}), 142.14-120.12$ (aromatic carbons), $75.54(\mathrm{~N}-\mathrm{C}-\mathrm{NH})$, $55.22(\mathrm{CH}), 33.22(\mathrm{CH}), 25.4\left(\mathrm{CH}_{2}\right), 15.3\left(\mathrm{CH}_{3}\right), 11.14\left(\mathrm{CH}_{3}\right)$ ppm. Anal.calcd for $\mathrm{C}_{15} \mathrm{H}_{19} \mathrm{~N}_{5} \mathrm{O}_{3}: \mathrm{C}, 56.77 ; \mathrm{H}, 6.03 ; \mathrm{N}, 22.07$. Found: C, 56.57; H, 6.06; N, 22.08.

2-(5-Amino-2-oxo-1, 2-dihydrospiro (indole-3, 3-(1, 2, 4) triazol)-4(2H)-yl)-3-methylbutanoic acid (5f). m.p. $195^{\circ} \mathrm{C}$; IR (KBr): 3433, 3155, 2988, 1709, 1678, 1595, 1533, 1427, $1094 \mathrm{~cm}^{-1}$; ${ }^{1} \mathrm{H}$ NMR $\left(\delta\right.$ ppm DMSO-d $\left.\mathrm{d}_{6}\right): 1.01\left(\mathrm{~d}, 3 \mathrm{H}, \mathrm{CH}_{3}\right), 1.03(\mathrm{~d}, 3 \mathrm{H}$, $\mathrm{CH}_{3}$ ), 2.1 (s, $2 \mathrm{H}, \mathrm{NH}_{2}$ of triazole ring), $2.39(\mathrm{~m}, 1 \mathrm{H}, \mathrm{CH})$, 3.49 (d, 1H, CH), 7.1 (s, 1H, N-H of triazole ring), 6.87-7.54 (m, $4 \mathrm{H}$, aromatic), 8.2 (s, $1 \mathrm{H}, \mathrm{NH}$ of indole ring), 11.2 (s, $1 \mathrm{H}$, $\mathrm{OH}) .{ }^{13} \mathrm{C}$ MR $\left(400 \mathrm{MHz}, \mathrm{DMSO}-\mathrm{d}_{6}\right): 174.12(\mathrm{C}=\mathrm{O}$, acid), 168.24 (C=O, indole), $162.16(\mathrm{C}=\mathrm{N}), 142.12-120.16$ (aromatic carbons), $75.58(\mathrm{~N}-\mathrm{C}-\mathrm{NH}), 58.28(\mathrm{CH}), 27.14\left(\mathrm{CH}_{2}\right), 17.14$ $\left(\mathrm{CH}_{3}\right), 17.12\left(\mathrm{CH}_{3}\right)$ ppm Anal.calcd for $\mathrm{C}_{14} \mathrm{H}_{17} \mathrm{~N}_{5} \mathrm{O}_{3}$ : C, 55.44; $\mathrm{H}, 5.65 ; \mathrm{N}, 23.09$. Found: C, 55.62; H, 5.68; N, 23.07.

2-(5-Amino-2-oxo-1, 2-dihydrospiro (indole-3, 3-(1, 2, 4) triazol)-4(2H)-yl)-3-hydroxypropanoic acid (5g). m.p. 90 C; IR (KBr): 3480, 3433, 3151, 2986, 1702, 1673, 1595, 1531, 1422, $1091 \mathrm{~cm}^{-1} ;{ }^{1} \mathrm{H}$ NMR $(\delta$ ppm DMSO-d 6$): 2.0(\mathrm{~s}, 1 \mathrm{H}, \mathrm{OH})$, $2.2\left(\mathrm{~s}, 2 \mathrm{H}, \mathrm{NH}_{2}\right.$ of triazole ring), $3.5(\mathrm{t}, 1 \mathrm{H}, \mathrm{CH}), 3.76(\mathrm{dd}$, $\left.2 \mathrm{H}, \mathrm{CH}_{2}\right), 7.0(\mathrm{~s}, 1 \mathrm{H}, \mathrm{N}-\mathrm{H}$ of triazole ring), 6.89-7.52 (m, $4 \mathrm{H}$, aromatic), $8.1(\mathrm{~s}, 1 \mathrm{H}, \mathrm{NH}$ of indole ring), $11.1(\mathrm{~s}, 1 \mathrm{H}$, $\mathrm{OH}) .{ }^{13} \mathrm{C}$ MR $\left(400 \mathrm{MHz}, \mathrm{DMSO}-\mathrm{d}_{6}\right): 174.12(\mathrm{C}=\mathrm{O}$, acid), $168.22(\mathrm{C}=\mathrm{O}$, indole), $162.16(\mathrm{C}=\mathrm{N}), 142.16-120.18$ (aromatic carbons), $75.54(\mathrm{~N}-\mathrm{C}-\mathrm{NH}), 58.22\left(\mathrm{CH}_{2}\right), 51.22(\mathrm{CH}) \mathrm{ppm}$. Anal.calcd for $\mathrm{C}_{12} \mathrm{H}_{13} \mathrm{~N}_{5} \mathrm{O}_{4}$ : C, 49.48; $\mathrm{H}, 4.50 ; \mathrm{N}, 24.04$. Found: C, 49.66; H, 4.52; N, 24.07.

2-(5-Amino-2-oxo-1, 2-dihydrospiro (indole-3, 3-(1, 2, 4) triazol)-4(2H)-yl)-3-phenylpropanoic acid (5h). m.p. 140-142 ${ }^{\circ} \mathrm{C}$;
IR (KBr): 3431, 3155, 2988, 1709, 1677, 1592, 1534, 1422, $1091 \mathrm{~cm}^{-1} ;{ }^{1} \mathrm{H}$ NMR $\left(\delta\right.$ ppm DMSO-d $\left.{ }_{6}\right): 2.3\left(\mathrm{~s}, 2 \mathrm{H}, \mathrm{NH}_{2}\right.$ of triazole ring), $2.78\left(\mathrm{dd}, 2 \mathrm{H}, \mathrm{CH}_{2}\right), 3.89(\mathrm{t}, 1 \mathrm{H}, \mathrm{CH}), 7.2(\mathrm{~s}, 1 \mathrm{H}$, $\mathrm{N}-\mathrm{H}$ of triazole ring), $6.88-7.52$ (m, $4 \mathrm{H}$, aromatic), 7.08-7.12 (m, $5 \mathrm{H}$, aromatic), 8.0 (s, $1 \mathrm{H}, \mathrm{NH}$ of indole ring), $11.0(\mathrm{~s}, 1 \mathrm{H}$, $\mathrm{OH}) .{ }^{13} \mathrm{CNMR}\left(400 \mathrm{MHz}, \mathrm{DMSO}-\mathrm{d}_{6}\right): 174.18(\mathrm{C}=\mathrm{O}$, acid), $168.26(\mathrm{C}=\mathrm{O}$, indole), $162.12(\mathrm{C}=\mathrm{N}), 142.14-122.14$ (aromatic carbons), $75.54(\mathrm{~N}-\mathrm{C}-\mathrm{NH}), 45.32(\mathrm{CH}), 34.22\left(\mathrm{CH}_{2}\right) \mathrm{ppm}$. Anal.calcd for $\mathrm{C}_{18} \mathrm{H}_{17} \mathrm{~N}_{5} \mathrm{O}_{3}$ : C, 61.53; $\mathrm{H}, 4.88 ; \mathrm{N}, 19.93$. Found: C, 61.33; H, 4.86; N, 19.95 .

2-(5-Amino-5-chloro-2-oxo-1, 2-dihydrospiro (indole-3, 3-(1, 2, 4) triazol)-4(2H)-yl)-3-phenylpropanoic acid (5i). m.p. $153^{\circ} \mathrm{C}$; IR (KBr): 3433, 3151, 2986, 1702, 1673, 1595, 1531, 1422, $1093 \mathrm{~cm}^{-1} ;{ }^{1} \mathrm{H}$ NMR $(\delta$ ppm DMSO-d $): 2.1\left(\mathrm{~s}, 2 \mathrm{H}, \mathrm{NH}_{2}\right.$ of triazole ring), $3.03\left(\mathrm{dd}, 2 \mathrm{H}, \mathrm{CH}_{2}\right), 3.88(\mathrm{t}, 1 \mathrm{H}, \mathrm{CH}), 7.3(\mathrm{~s}, 1 \mathrm{H}$, $\mathrm{N}-\mathrm{H}$ of triazole ring), 7.08-7.21 (m, 5H, aromatic), 7.05-7.46 (m, 3H, aromatic), 8.2 (s, $1 \mathrm{H}, \mathrm{NH}$ of indole ring), 11.1 (s, $1 \mathrm{H}$, $\mathrm{OH}) .{ }^{13} \mathrm{CNMR}\left(400 \mathrm{MHz}, \mathrm{DMSO}-\mathrm{d}_{6}\right): 174.18$ (C=O, acid), $168.26(\mathrm{C}=\mathrm{O}$, indole), $162.12(\mathrm{C}=\mathrm{N}), 142.14-120.12$ (aromatic carbons), $75.54(\mathrm{~N}-\mathrm{C}-\mathrm{NH}), 45.38(\mathrm{CH}), 34.22\left(\mathrm{CH}_{2}\right) \mathrm{ppm}$. Anal.calcd for $\mathrm{C}_{18} \mathrm{H}_{16} \mathrm{~N}_{5} \mathrm{O}_{3} \mathrm{Cl}$ : C, 56.04; H, 4.18; N, 18.15 . Found: C, 56.24; H, 4.21; N, 18.17 .

2-(5-Amino-5-chloro-2-oxo-1, 2-dihydrospiro (indole-3, 3-(1, 2 , 4) triazol)-4(2H)-yl)-3-(1H-imidazole-4-yl)propanoic acid (5j). m.p. $180-182^{\circ} \mathrm{C}$; IR (KBr): 3431, 3155, 2989, 1707, 1676, $1598,1530,1428,1091,721 \mathrm{~cm}^{-1} ;{ }^{1} \mathrm{H}$ NMR $(\delta$ ppm DMSO-d 6 ): $2.0\left(\mathrm{~s}, 2 \mathrm{H}, \mathrm{NH}_{2}\right.$ of triazole ring), $3.04\left(\mathrm{dd}, 2 \mathrm{H}, \mathrm{CH}_{2}\right), 3.89(\mathrm{t}$, $1 \mathrm{H}, \mathrm{CH}), 6.80(\mathrm{~d}, 1 \mathrm{H}, \mathrm{CH}), 7.1(\mathrm{~s}, 1 \mathrm{H}, \mathrm{N}-\mathrm{H}$ of triazole ring), 7.44 (d, 1H, CH), 7.05-7.47 (m, 3H, aromatic), 8.1 (s, 1H, NH of indole ring), $11.1(\mathrm{~s}, 1 \mathrm{H}, \mathrm{OH}), 13.4(\mathrm{t}, 1 \mathrm{H}, \mathrm{NH}) .{ }^{13} \mathrm{CNMR}$ (400 MHz, DMSO-d $\left.)_{6}\right): 174.18(\mathrm{C}=\mathrm{O}$, acid), $168.26(\mathrm{C}=\mathrm{O}$, indole), $162.12(\mathrm{C}=\mathrm{N}), 142.14-120.12$ (aromatic carbons), $75.54(\mathrm{~N}-\mathrm{C}-\mathrm{NH}), 45.38(\mathrm{CH}), 27.12\left(\mathrm{CH}_{2}\right)$ ppm. Anal.calcd for $\mathrm{C}_{15} \mathrm{H}_{14} \mathrm{~N}_{7} \mathrm{O}_{3} \mathrm{Cl}$ : C, 47.94; $\mathrm{H}, 3.76 ; \mathrm{N}, 26.09$. Found: $\mathrm{C}$, 47.74; H, 3.78; N, 26.12.

2-(5-Amino-5-chloro-2-oxo-1, 2-dihydrospiro (indole-3, 3-(1, 2, 4) triazol)-4(2H)-yl)-5-(methylsulfanyl) butanoic acid acid (5k). m.p. $220^{\circ}$ C; IR (KBr): 3434, 3152, 2982, 1701, 1672, 1597, $1532,1426,1095,726 \mathrm{~cm}^{-1} ;{ }^{1} \mathrm{H}$ NMR $(\delta$ ppm DMSO-d 6 ): 2.1 (s, $2 \mathrm{H}, \mathrm{NH}_{2}$ of triazole ring), $2.01\left(\mathrm{q}, 2 \mathrm{H}, \mathrm{CH}_{2}\right), 2.08(\mathrm{~s}, 3 \mathrm{H}$, $\mathrm{CH}_{3}$ ), 2.43 (t, 2H, $\mathrm{CH}_{2}$ ), 3.49 (t, 1H, CH), 7.0 (s, 1H, N-H of triazole ring), 7.05-7.46 (m, 3H, aromatic), $8.0(\mathrm{~s}, 1 \mathrm{H}, \mathrm{NH}$ of indole ring), $10.1(\mathrm{t}, 1 \mathrm{H}, \mathrm{NH}) .11 .1(\mathrm{~s}, 1 \mathrm{H}, \mathrm{OH}) .{ }^{13} \mathrm{CNMR}$ (400 MHz, DMSO-d $\left.\mathrm{d}_{6}\right): 174.12(\mathrm{C}=\mathrm{O}$, acid), $168.22(\mathrm{C}=\mathrm{O}$, indole), $162.18(\mathrm{C}=\mathrm{N}), 142.18-120.18$ (aromatic carbons), $75.58(\mathrm{~N}-\mathrm{C}-\mathrm{NH}), 51.22(\mathrm{CH}), 27.12\left(\mathrm{CH}_{2}\right), 25.14\left(\mathrm{CH}_{2}\right), 17.14$ $\left(\mathrm{CH}_{3}\right)$ ppm. Anal.calcd for $\mathrm{C}_{15} \mathrm{H}_{18} \mathrm{~N}_{5} \mathrm{O}_{3} \mathrm{SCl}$ : C, 46.93; $\mathrm{H}$, 4.73; N, 18.24. Found: C, 46.73; H, 4.70; N, 18.27.

2-(5-Amino-5-chloro-2-oxo-1, 2-dihydrospiro (indole-3, 3-(1, 2, 4) triazol)-4(2H)-yl)-3-(2,3-dihydro-1H-indole-3-yl)propanoic acid (5l). m.p. $230^{\circ} \mathrm{C}$; IR (KBr): 3431, 3150, 2980, 1703, 1670, $1595,1531,1424,1097,729 \mathrm{~cm}^{-1} ;{ }^{1} \mathrm{H}$ NMR $(\delta$ ppm DMSO-d 6 ): $1.93\left(\mathrm{t}, 2 \mathrm{H}, \mathrm{CH}_{2}\right), 2.2\left(\mathrm{~s}, 2 \mathrm{H}, \mathrm{NH}_{2}\right.$ of triazole ring), $3.00(\mathrm{t}$, $1 \mathrm{H}, \mathrm{CH}), 3.22\left(\mathrm{dd}, 2 \mathrm{H}, \mathrm{CH}_{2}\right), 3.48(\mathrm{t}, 1 \mathrm{H}, \mathrm{CH}), 7.1(\mathrm{~s}, 1 \mathrm{H}$, 
$\mathrm{N}-\mathrm{H}$ of triazole ring), 6.35-6.91 (m, 4H, aromatic), 7.06$7.46(\mathrm{~m}, 3 \mathrm{H}$, aromatic), $8.3(\mathrm{~s}, 1 \mathrm{H}, \mathrm{NH}$ of indole ring), 10.1 (s, 1H, NH), 11.1 (s, 1H, OH). ${ }^{13} \mathrm{C}$ NMR $(400 \mathrm{MHz}, \mathrm{DMSO}-$ $\left.\mathrm{d}_{6}\right)$ : $174.18(\mathrm{C}=\mathrm{O}$, acid), $168.26(\mathrm{C}=\mathrm{O}$, indole $), 162.12(\mathrm{C}=\mathrm{N})$, 142.14-120.12 (aromatic carbons), 75.54 (N-C-NH), 49.32 $(\mathrm{CH}), 32.12\left(\mathrm{CH}_{2}\right)$ ppm. Anal.calcd for $\mathrm{C}_{20} \mathrm{H}_{19} \mathrm{~N}_{6} \mathrm{O}_{3} \mathrm{Cl}: \mathrm{C}$, 56.28; H, 4.49; N, 19.69. Found: C, 56.48; H, 4.46; N, 19.66.

2-(5-Amino-5-nitro-2-oxo-1, 2-dihydrospiro (indole-3, 3-(1, 2, 4) triazol)-4(2H)-yl)-3-phenylpropanoic acid (5m). m.p. $210^{\circ} \mathrm{C}$; IR (KBr): 3433, 3152, 2982, 1701, 1675, 1591, 1532, 1422, $1365,1092 \mathrm{~cm}^{-1} ;{ }^{1} \mathrm{H}$ NMR $(\delta$ ppm DMSO-d 6$): 2.1\left(\mathrm{~s}, 2 \mathrm{H}, \mathrm{NH}_{2}\right.$ of triazole ring), $3.03\left(\mathrm{dd}, 2 \mathrm{H}, \mathrm{CH}_{2}\right), 3.88(\mathrm{t}, 1 \mathrm{H}, \mathrm{CH}), 7.3$ (s, $1 \mathrm{H}, \mathrm{N}-\mathrm{H}$ of triazole ring), $7.08-7.21(\mathrm{~m}, 5 \mathrm{H}$, aromatic), 7.09-7.46 ( $\mathrm{m}, 3 \mathrm{H}$, aromatic), $8.2(\mathrm{~s}, 1 \mathrm{H}, \mathrm{NH}$ of indole ring), $11.1(\mathrm{~s}, 1 \mathrm{H}, \mathrm{OH}) .{ }^{13} \mathrm{CNMR}\left(400 \mathrm{MHz}, \mathrm{DMSO}-\mathrm{d}_{6}\right): 174.12$ $(\mathrm{C}=\mathrm{O}$, acid $), 168.24(\mathrm{C}=\mathrm{O}$, indole $), 162.14(\mathrm{C}=\mathrm{N}), 142.12-$ 120.10 (aromatic carbons), $75.52(\mathrm{~N}-\mathrm{C}-\mathrm{NH}), 45.34(\mathrm{CH})$, $34.28\left(\mathrm{CH}_{2}\right)$ ppm. Anal.calcd for $\mathrm{C}_{18} \mathrm{H}_{16} \mathrm{~N}_{6} \mathrm{O}_{5}: \mathrm{C}, 54.54 ; \mathrm{H}$, 4.07; N, 21.20. Found: C, 54.37; H, 4.09; N, 21.21.

2-(5-Amino-5-nitro-2-oxo-1, 2-dihydrospiro (indole-3, 3-(1, 2, 4) triazol)-4(2H)-yl)-3-(1H-imidazole-4-yl)propanoic acid (5n). m.p. $207^{\circ}$ C; IR (KBr): 3431, 3155, 2986, 1704, 1672, 1594, $1536,1422,13661092 \mathrm{~cm}^{-1} ;{ }^{1} \mathrm{H}$ NMR $(\delta$ ppm DMSO-d 6 ): 2.0 (s, $2 \mathrm{H}, \mathrm{NH}_{2}$ of triazole ring), $3.04\left(\mathrm{dd}, 2 \mathrm{H}, \mathrm{CH}_{2}\right), 3.89(\mathrm{t}, 1 \mathrm{H}$, $\mathrm{CH}), 6.80(\mathrm{~d}, 1 \mathrm{H}, \mathrm{CH}), 7.1$ (s, 1H, N-H of triazole ring), 7.44 (d, 1H, CH), 7.04-7.45 (m, 3H, aromatic), 8.1 (s, 1H, NH of indole ring), $11.3(\mathrm{~s}, 1 \mathrm{H}, \mathrm{OH}), 13.4(\mathrm{t}, 1 \mathrm{H}, \mathrm{NH}) .{ }^{13} \mathrm{CNMR}$ $\left(400 \mathrm{MHz}, \mathrm{DMSO}-\mathrm{d}_{6}\right): 174.14(\mathrm{C}=\mathrm{O}$, acid $), 168.26(\mathrm{C}=\mathrm{O}$, indole), $162.12(\mathrm{C}=\mathrm{N}), 142.18-120.12$ (aromatic carbons), $75.52(\mathrm{~N}-\mathrm{C}-\mathrm{NH}), 45.35(\mathrm{CH}), 27.22\left(\mathrm{CH}_{2}\right)$ ppm Anal.calcd for $\mathrm{C}_{15} \mathrm{H}_{14} \mathrm{~N}_{8} \mathrm{O}_{5}$ : C, 46.63; H, 3.65; N, 29.01. Found: C, 46.43; $\mathrm{H}, 3.67$; N, 29.03 .

2-(5-Amino-5-nitro-2-oxo-1, 2-dihydrospiro (indole-3, 3-(1, 2, 4) triazol)-4(2H)-yl)-5-(methylsulfanyl)butanoic acid (5o). m.p. $194^{\circ} \mathrm{C}$; IR (KBr): 3434, 3153, 2984, 1702, 1672, 1599, 1533, 1421, $13621097 \mathrm{~cm}^{-1} ;{ }^{1} \mathrm{H}$ NMR ( $\delta$ ppm DMSO-d 6 ): $1.80(\mathrm{q}$, $\left.2 \mathrm{H}, \mathrm{CH}_{2}\right), 2.10\left(\mathrm{~s}, 2 \mathrm{H}, \mathrm{NH}_{2}\right.$ of triazole ring), $2.30(\mathrm{~s}, 3 \mathrm{H}$, $\left.\mathrm{CH}_{3}\right), 2.50\left(\mathrm{t}, 2 \mathrm{H}, \mathrm{CH}_{2}\right), 3.49(\mathrm{t}, 1 \mathrm{H}, \mathrm{CH}), 7.1(\mathrm{~s}, 1 \mathrm{H}, \mathrm{N}-$ $\mathrm{H}$ of triazole ring), $7.78-7.98(\mathrm{~m}, 3 \mathrm{H}$, aromatic), $8.2(\mathrm{~s}, 1 \mathrm{H}$, $\mathrm{NH}$ of indole ring), $11.1(\mathrm{~s}, 1 \mathrm{H}, \mathrm{OH}) .{ }^{13} \mathrm{CNMR}(400 \mathrm{MHz}$, DMSO): 174.18 ( $\mathrm{C}=\mathrm{O}$, acid), 168.22 ( $\mathrm{C}=\mathrm{O}$, indole), 162.12 $(\mathrm{C}=\mathrm{N}), 142.14-132.76$ (aromatic carbons), $75.54(\mathrm{~N}-\mathrm{C}-\mathrm{NH})$, $51.22(\mathrm{CH}), 35.02\left(\mathrm{CH}_{2}\right), 27.14\left(\mathrm{CH}_{2}\right), 25.12\left(\mathrm{CH}_{2}\right), 17.64$ $\left(\mathrm{CH}_{3}\right)$ ppm. Anal.calcd for $\mathrm{C}_{15} \mathrm{H}_{18} \mathrm{~N}_{6} \mathrm{O}_{5} \mathrm{~S}: \mathrm{C}, 45.68 ; \mathrm{H}, 4.60$; N, 21.31. Found: C, 44.40; H, 4.26; N, 22.07.

2-(5-Amino-5-nitro-2-oxo-1, 2-dihydrospiro (indole-3, 3-(1, 2, 4) triazol)-4(2H)-yl)-3-(2,3-dihydro-1H-indole-3-yl)propanoic acid (5p). m.p. $185^{\circ} \mathrm{C}$; IR (KBr): 3431, 3152, 2982, 1706, 1678, 1594, 1531, 1429, $13651093 \mathrm{~cm}^{-1}$; ${ }^{1} \mathrm{H}$ NMR ( $\delta$ ppm DMSO$\left.\mathrm{d}_{6}\right): 1.93\left(\mathrm{t}, 2 \mathrm{H}, \mathrm{CH}_{2}\right), 2.2\left(\mathrm{~s}, 2 \mathrm{H}, \mathrm{NH}_{2}\right.$ of triazole ring), 3.00 $(\mathrm{t}, 1 \mathrm{H}, \mathrm{CH}), 3.47$ (dd, $\left.2 \mathrm{H}, \mathrm{CH}_{2}\right), 3.60(\mathrm{t}, 1 \mathrm{H}, \mathrm{CH}), 7.1(\mathrm{~s}, 1 \mathrm{H}$, $\mathrm{N}-\mathrm{H}$ of triazole ring), 6.35-6.91 (m, $4 \mathrm{H}$, aromatic), 7.78-7.98 ( $\mathrm{m}, 3 \mathrm{H}$, aromatic), 8.1 (s, $1 \mathrm{H}, \mathrm{NH}$ of indole ring), $10.1(\mathrm{~s}, 1 \mathrm{H}$, $\mathrm{NH}), 11.1$ (s, 1H, OH). ${ }^{13} \mathrm{C}$ NMR (400 MHz, DMSO): 174.18
$(\mathrm{C}=\mathrm{O}$, acid $), 168.26(\mathrm{C}=\mathrm{O}$, indole $), 162.12(\mathrm{C}=\mathrm{N}), 142.14-$ 117.14 (aromatic carbons), $75.54(\mathrm{~N}-\mathrm{C}-\mathrm{NH}), 49.32(\mathrm{CH}), 32.12$ $\left(\mathrm{CH}_{2}\right)$ ppm. Anal.calcd for $\mathrm{C}_{20} \mathrm{H}_{19} \mathrm{~N}_{7} \mathrm{O}_{5}: \mathrm{C}, 54.92 ; \mathrm{H}, 4.38 ; \mathrm{N}$, 22.42. Found: C, 44.40; H, 4.26; N, 22.07.

\section{Conclusion}

The use of water as a green solvent offers a convenient, nontoxic, and inexpensive reaction medium for the environeconomic synthesis of triazole derivatives. This procedure is simpler, economical, milder, and faster, including cleaner reactions, high yields of products, and a simple experimental and work-up procedure, which makes it a useful and attractive process and is also consistent with the green chemistry theme which affords good yields. Synthesized compounds are found to be excellent fluorescent materials and potent fungicidal agents.

\section{Acknowledgments}

The authors are thankful to the Dean of FET, MITS, for providing necessary research facilities in the department. Financial assistance from FET, MITS is gratefully acknowledged. They are also thankful to SAIF Punjab University, Chandigarh, for the spectral and elemental analyses.

\section{References}

[1] T. J. J. Müller, "Multicomponent reactions," Beilstein Journal of Organic Chemistry, vol. 7, pp. 960-961, 2011.

[2] R. P. Tenório, C. S. Carvalho, C. S. Pessanha et al., "Synthesis of thiosemicarbazone and 4-thiazolidinone derivatives and their in vitro anti-Toxoplasma gondii activity," Bioorganic \& Medicinal Chemistry Letters, vol. 15, pp. 2575-2578, 2005.

[3] M. C. Pirrung, S. V. Pansare, K. Das Sarma, K. A. Keith, and E. R. Kern, "Combinatorial optimization of isatin- $\beta$-thiosemicarbazones as anti-poxvirus agents," Journal of Medicinal Chemistry, vol. 48, no. 8, pp. 3045-3050, 2005.

[4] W.-X. Hu, W. Zhou, C.-N. Xia, and X. Wen, "Synthesis and anticancer activity of thiosemicarbazones," Bioorganic \& Medicinal Chemistry Letters, vol. 16, pp. 2213-2218, 2006.

[5] R. B. de Oliveira, E. M. de Souza-Fagundes, R. P. P. Soares, A. A. Andrade, A. U. Kretti, and C. L. Zani, "Synthesis and antimalarial activity of semicarbazone and thiosemicarbazone derivatives," European Journal of Medicinal Chemistry, vol. 43, pp. 183-188, 2008.

[6] A. Pérez-Rebolledo, L. R. Teixeira, A. A. Batista et al., "4Nitroacetophenone-derived thiosemicarbazones and their copper(II) complexes with significant in vitro anti-trypanosomal activity," European Journal of Medicinal Chemistry, vol. 43, pp. 939-948, 2008.

[7] G. Aguirre, L. Boiani, H. Cerecetto et al., "In vitro activity and mechanism of action against the protozoan parasite Trypanosoma cruzi of 5-nitrofuryl containing thiosemicarbazones," Bioorganic and Medicinal Chemistry, vol. 12, no. 18, pp. 48854893, 2004.

[8] X. Du, C. Guo, E. Hansell et al., "Synthesis and structure-activity relationship study of potent trypanocidal thio semicarbazone inhibitors of the trypanosomal cysteine protease cruzain," 
Journal of Medicinal Chemistry, vol. 45, no. 13, pp. 2695-2707, 2002.

[9] N. Fujii, J. P. Mallari, E. J. Hansell et al., "Discovery of potent thiosemicarbazone inhibitors of rhodesain and cruzain," Bioorganic and Medicinal Chemistry Letters, vol. 15, no. 1, pp. 121-123, 2005.

[10] R. P. Tenório, A. J. S. Góes, J. G. de Lima, A. R. de Faria, A. J. Alves, and T. M. Aquino, "Thiosemicarbazones: preparation methods, synthetic applications and biological importance," Química Nova, vol. 28, pp. 1030-1037, 2005.

[11] J. M. Kane, B. M. Baron, M. W. Dudley, S. M. Sorensen, M. A. Staeger, and F. P. Miller, "2,4-Dihydro-3H-1,2,4-triazol-3-ones as anticonvulsant agents," Journal of Medicinal Chemistry, vol. 33, no. 10, pp. 2772-2777, 1990.

[12] S. Rollas, N. Kalyoncuoglu, D. Sur-Altiner, and Y. Yegenoglu, "5-(4-Aminophenyl)-4-substituted-2,4-dihydro-3H-1,2,4triazole-3-thione s: synthesis and antibacterial and antifungal activities," Pharmazie, vol. 48, no. 4, pp. 308-309, 1993.

[13] B. E. Gilbert and V. Knight, "Biochemistry and clinical applications of ribavirin," Antimicrobial Agents and Chemotherapy, vol. 30, no. 2, p. 201, 1986.

[14] P. C. Wade, B. Richard Vogt, T. P. Kissick, L. M. Simpkins, D. M. Palmer, and R. C. Millonig, "1-Acyltriazoles as antiinflammatory agents," Journal of Medicinal Chemistry, vol. 25, no. 3, pp. 331-333, 1982.

[15] F. Malbec, R. Milcent, P. Vicart, and A. M. Bure, "Synthesis of new derivatives of 4-amino-2,4-dihydro-1,2,4-triazol-3-one as potential antibacterial agents," Journal of Heterocyclic Chemistry, vol. 21, no. 6, pp. 1769-1774, 1984.

[16] L. I. Kruse, D. L. Ladd, P. B. Harrsch et al., "Synthesis, tubulin binding, antineoplastic evaluation, and structure-activity relationship of oncodazole analogues," Journal of Medicinal Chemistry, vol. 32, no. 2, pp. 409-417, 1989.

[17] S. Ram, D. S. Wise, L. L. Wotring, J. W. McCall, and L. B. Townsend, "Synthesis and biological activity of certain alkyl 5(alkoxycarbonyl)-1H-benzimidazole-2-carbamates and related derivatives: a new class of potential antineoplastic and antifilarial agents," Journal of Medicinal Chemistry, vol. 35, no. 3, pp. 539-547, 1992.

[18] E. R. Cole, G. Crank, and A. Salam-Sheikh, "Antioxidant properties of benzimidazoles." Journal of Agricultural and Food Chemistry, vol. 22, no. 5, p. 918, 1974.

[19] C. Hubschwerlen, P. Pflieger, J. L. Specklin et al., "Pyrimido[1,6a]benzimidazoles: a new class of DNA gyrase inhibitors," Journal of Medicinal Chemistry, vol. 35, no. 8, pp. 1385-1392, 1992.

[20] P. Venkatesan, “Albendazole," Journal of Antimicrobial Chemotherapy, vol. 41, pp. 145-147, 1998.

[21] N. S. Pawar, D. S. Dalal, S. R. Shimpi, and P. P. Mahulikar, "Studies of antimicrobial activity of $\mathrm{N}$-alkyl and $\mathrm{N}$-acyl 2-(4thiazolyl)-1H-benzimidazoles ," European Journal of Pharmaceutical Sciences, vol. 21, pp. 115-118, 2004.

[22] T. Ishida, T. Suzuki, S. Hirashima et al., "Benzimidazole inhibitors of hepatitis C virus NS5B polymerase: identification of 2-[(4-diarylmethoxy)phenyl]-benzimidazole," Bioorganic and Medicinal Chemistry Letters, vol. 16, pp. 1859-1863, 2006.

[23] L. Garuti, M. Roberti, and G. Gentilomi, "Synthesis and antiviral assays of some benzimidazole nucleosides and acyclonucleosides," Farmaco, vol. 56, no. 11, pp. 815-819, 2001.

[24] K. Kubo, Y. Kohara, Y. Yoshimura et al., "Nonpeptide angiotensin II receptor antagonists. Synthesis and biological activity of potential prodrugs of benzimidazole-7-carboxylic acids,"
Journal of Medicinal Chemistry, vol. 36, no. 16, pp. 2343-2349, 1993.

[25] K. Stratmann, R. E. Moore, R. Bonjouklian et al., "Welwitindolinones, unusual alkaloids from the blue-green algae Hapalosiphon welwitschii and Westiella intricata. Relationship to fischerindoles and hapalindoles," Journal of the American Chemical Society, vol. 116, no. 22, pp. 9935-9942, 1994.

[26] J. W. Skiles and D. Menil, "Spiro indolinone beta-lactams, inhibitors of poliovirus and rhinovlrus 3C-proteinases," Tetrahedron Letters, vol. 31, p. 7277, 1990.

[27] A. Hasan, N. F. Thomas, and S. Gapil, "Synthesis, characterization and antifungal evaluation of 5-substituted-4-amino-1,2,4triazole-3-thioesters," Molecules, vol. 16, no. 2, pp. 1297-1309, 2011.

[28] S. Veda and H. Nagasawa, "Facile synthesis of 1,2,4-triazoles via a copper-catalyzed tandem addition-oxidative cyclization," Journal of the American Chemical Society, vol. 131, pp. 1508015081, 2009.

[29] G. M. Castanedo, P. S. Seng, N. Blaquiere, S. Trapp, and S. T. Staben, "Rapid synthesis of 1,3,5-substituted 1,2,4-triazoles from carboxylic acids, amidines, and hydrazines," Journal of Organic Chemistry, vol. 76, no. 4, pp. 1177-1179, 2011.

[30] Y. Xu, M. Mc Laughlin, E. N. Bolton, and R. A. Reamer, "Practical synthesis of functionalized 1,5-disubstituted 1,2,4triazole derivatives," Journal of Organic Chemistry, vol. 75, pp. 8666-8669, 2010.

[31] A. Dandia, P. Sarawgi, K. Arya, and S. Khaturia, "Mild and ecofriendly tandem synthesis of 1,2,4-triazolo[4,3-a]pyrimidines in aqueous medium ", Arkivoc, no. 16, pp. 83-92, 2006.

[32] A. Jha, Y. L. N. Murthy, G. Durga, and T. T. Sundari, "Microwave-assisted synthesis of 3,5-Dibenzyl-4-amino-1,2,4-triazole and its diazo ligand, metal complexes along with anticancer activity," E-Journal of Chemistry, vol. 7, no. 4, pp. 1571-1577, 2010.

[33] A. Dandia, H. Sachdeva, and R. Singh, "Montmorillonite catalysed synthesis of novel spiro[3H-indole-3,3'-[3H-1,2,4] triazol]-2(1H) ones in dry media under microwave irradiation," Journal of Chemical Research, vol. 2000, no. 6, pp. 272-275, 2000.

[34] A. Dandia, R. Singh, S. Bhaskaran, and D. S. Shriniwas, "Versatile three-component procedure for combinatorial synthesis of biologically relevant scaffold spiro[indole-thiazolidinones] under aqueous conditions," Green Chemistry, vol. 13, pp. 18521859, 2011.

[35] T. K. Huang, R. Wang, S. Lin, and X. Lu, "Montmorillonite K-10: an efficient and reusable catalyst for the synthesis of quinoxaline derivatives in water," Catalysis Communications, vol. 9, pp. 11431147, 2008.

[36] G. P. Chiusoli, M. Costa, L. Cucchia, B. Gabriele, G. Salerno, and L. Veltri, "Carbon dioxide effect on palladium-catalyzed sequential reactions with carbon monoxide, acetylenic compounds and water," Journal of Molecular Catalysis A, vol. 204205, pp. 133-142, 2003.

[37] M. Lautens and M. Yoshida, "Rhodium-catalyzed addition of arylboronic acids to alkynyl aza-heteroaromatic compounds in water," Journal of Organic Chemistry, vol. 68, p. 762, 2003.

[38] H. Kim, S. Lee, J. Lee, and J. Tae, "Rhodamine triazole-based fluorescent probe for the detection of $\mathrm{Pt}^{2+}$," Organic Letters, vol. 12, no. 22, pp. 5342-5345, 2010.

[39] S. Y. Park, J. H. Yoon, C. S. Hong et al., "A pyrenyl-appended triazole-based calix[4] arene as a fluorescent sensor for $\mathrm{Cd}^{2+}$ and $\mathrm{Zn}^{2+}$," Journal of Organic Chemistry, vol. 73, no. 21, pp. 82128218, 2008. 
[40] J. D. Cheon, T. Mutai, and K. Araki, "Preparation of a series of novel fluorophores, N-substituted 6-amino and 6,6" diamino-2,2' $: 6^{\prime}, 2^{\prime \prime}$-terpyridine by palladium-catalyzed amination," Tetrahedron Letters, vol. 47, no. 29, pp. 5079-5082, 2006.

[41] S. Coe, W. K. Woo, M. Bawendi, and V. Bulović, "Electroluminescence from single monolayers of nanocrystals in molecular organic devices," Nature, vol. 420, no. 6917, pp. 800-803, 2002.

[42] G. Hughes and M. R. Bryce, "Electron-transporting materials for organic electroluminescent and electrophosphorescent devices," Journal of Materials Chemistry, vol. 15, pp. 94-107, 2005.

[43] M. R. Detty, S. L. Gibson, and S. J. Wagner, "Current clinical and preclinical photosensitizers for use in photodynamic therapy," Journal of Medicinal Chemistry, vol. 47, no. 16, pp. 3897-3915, 2004.

[44] H. Sachdeva, D. Dwivedi, R. R. Bhattacharjee, S. Khaturia, and R. Saroj, "NiO nanoparticles: an efficient catalyst for the multicomponent one-pot synthesis of novel spiro and condensed indole derivatives," Journal of Chemistry, vol. 2013, Article ID 606259, 10 pages, 2013.

[45] H. Sachdeva and D. Dwivedi, "Lithium-acetate-mediated biginelli one-pot multicomponent synthesis under solvent-free conditions and cytotoxic activity against the human lung cancer cell line A549 and breast cancer cell line MCF7," The Scientific World Journal, vol. 2012, Article ID 109432, 9 pages, 2012.

[46] H. Sachdeva, R. Saroj, S. Khaturia, and D. Dwivedi, "Operationally simple green synthesis of some Schiff bases using grinding chemistry technique and evaluation of antimicrobial activities," Green Processing and Synthesis, vol. 1, pp. 469-477, 2012.

[47] H. Sachdeva, D. Dwivedi, and S. Khaturia, "Aqua mediated facile synthesis of 2-(5/7-fluorinated-2-oxoindolin-3-ylidene)$N$-(4-substituted phenyl) hydrazine carbothioamides," Research Journal of Pharmaceutical, Biological and Chemical Science, vol. 2, no. 2, p. 213, 2011.

[48] H. Sachdeva, R. Saroj, S. Khaturia, and H. L. Singh, "Comparative studies of lewis acidity of alkyl-tin chlorides in multicomponent biginelli condensation using grindstone chemistry technique," Journal of the Chilean Chemical Society, vol. 57, no. 1, p. 1012, 2012.

[49] S. Patil, S. D. Jadhav, and U. P. Patil, "Natural acid catalyzed synthesis of schiff base under solvent-free condition: as a green approach," Archives of Applied Science Research, vol. 4, no. 2, pp. 1074-1078, 2012.

[50] S. Patil, S. D. Jadhav, and M. B. Deshmukh, "Natural acid catalyzed synthesis of schiff base under solvent-free condition: as a green approach," Archives of Applied Science Research, vol. 3, no. 1, pp. 203-208, 2011. 

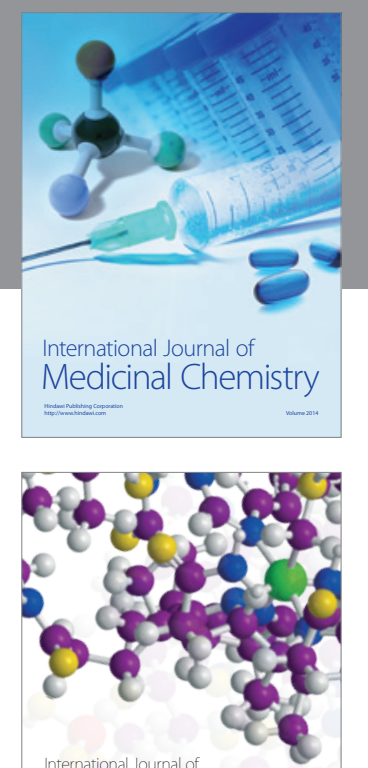

\section{Carbohydrate} Chemistry

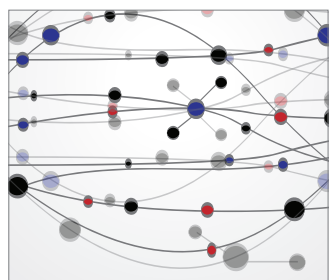

The Scientific World Journal
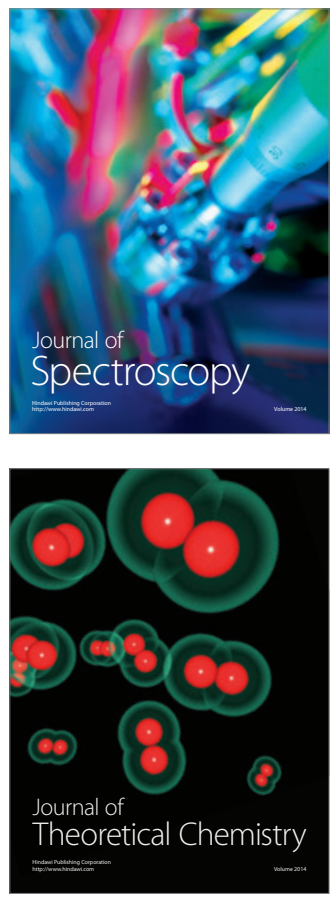
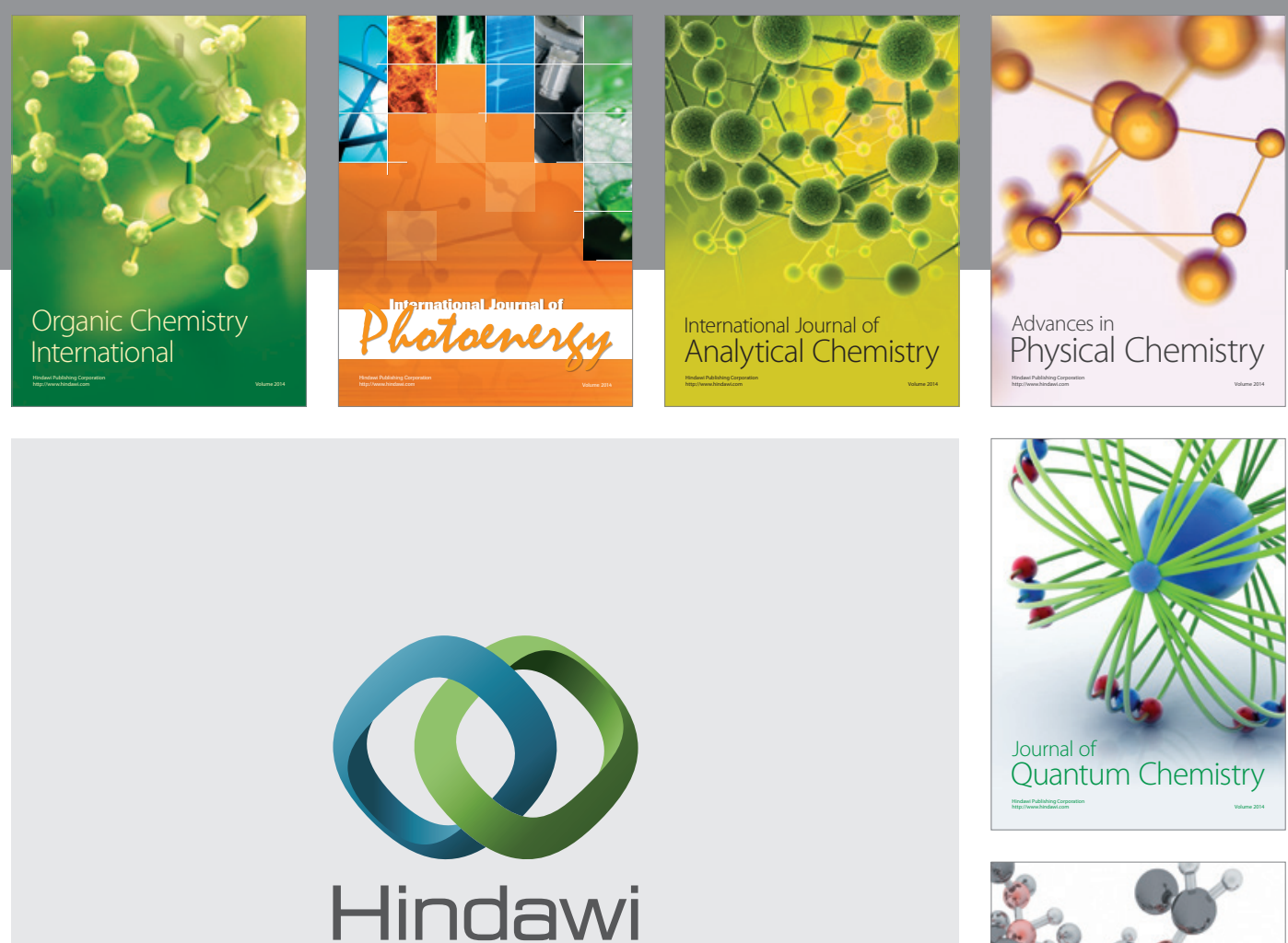

Submit your manuscripts at

http://www.hindawi.com

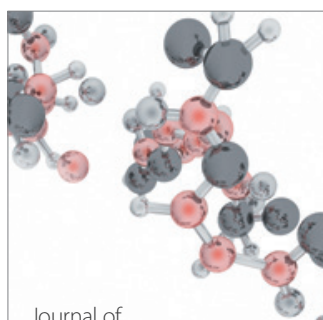

Analytical Methods

in Chemistry

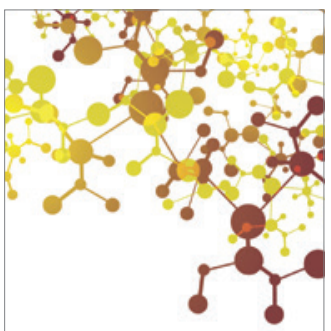

Journal of

Applied Chemistry

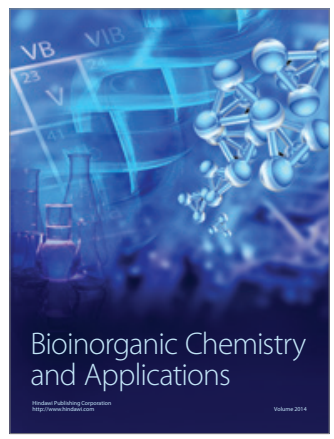

Inorganic Chemistry
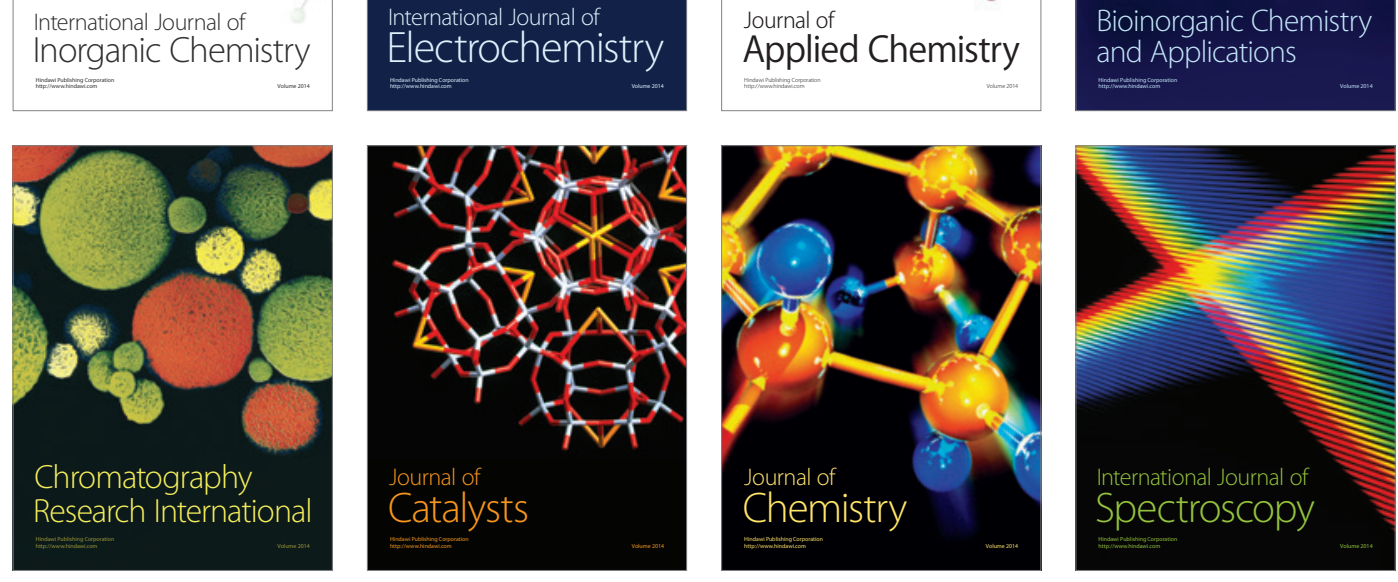